\title{
Conciliatory reasoning, self-defeat, and abstract argumentation*
}

\author{
Aleks Knoks \\ University of Luxembourg
}

\begin{abstract}
According to conciliatory views on the significance of disagreement, it's rational for you to become less confident in your take on an issue in case your epistemic peer's take on it is different. These views are intuitively appealing, but they also face a powerful objection: in scenarios that involve disagreements over their own correctness, conciliatory views appear to self-defeat and, thereby, issue inconsistent recommendations. This paper provides a response to this objection. Drawing on the work from defeasible logics paradigm and abstract argumentation, it develops a formal model of conciliatory reasoning and explores its behavior in the troubling scenarios. The model suggests that the recommendations that conciliatory views issue in such scenarios are perfectly reasonable - even if outwardly they may look odd.
\end{abstract}

Keywords: disagreement, conciliationism, self-defeat, abstract argumentation theory, defeasible logic

\section{Contents}

1 Introduction 1

2 Conciliatory reasoning in default logic 4

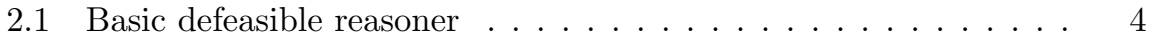

2.2 Capturing conciliationism . . . . . . . . . . . . . . 8

2.3 Disagreements over disagreement . . . . . . . . . . . . . 11

3 Moving to argumentation theory $\quad 17$

3.1 Argument frameworks . . . . . . . . . . . . . . 18

3.2 Selecting winning arguments . . . . . . . . . . . . . . . 20

3.3 Minimal arguments and basic defeat . . . . . . . . . . . . . 24

3.4 Disagreements over disagreement revisited . . . . . . . . . . . . 27

${ }^{*}$ Forthcoming in Review of Symbolic Logic. Please, cite the published version once it becomes available. 
4 Adding degrees of confidence $\quad 31$

4.1 Relativizing support to degrees of support . . . . . . . . . . . . 31

4.2 From degrees of confidence to degrees of support . . . . . . . . . . 36

4.3 Disagreements over disagreement with degrees . . . . . . . . . 40

5 Conclusion

Appendix: Proofs of the central observations 


\section{Introduction}

Think of your favorite philosophical problem. You've likely thought about it for a long time, and you must have some idea regarding how to resolve it. And odds are you know someone who has thought about it for at least as long, whose credential are as good as yours, and whose solution to the problem is incompatible with yours. If so, you're in disagreement with an epistemic peer. ${ }^{1}$ Should this fact make you less confident that your solution to the problem is the correct one? According to the so-called conciliatory or conciliationist views, it should. This answer has much intuitive appeal: the problem is complex, you are not infallible, and one straightforward explanation for the disagreement is that you've made a subtle mistake when reasoning. An equally good explanation is that your opponent has made a mistake. But given that there's no good reason to favor the latter, reducing confidence still seems appropriate. ${ }^{2}$

But their intuitive appeal notwithstanding, conciliatory views are said to run into problems when applied to themselves, or when answering the question of what should one do when disagreeing about the epistemic significance of disagreement. The problems are most transparent and easiest to explain for the more extreme conciliatory views. According to such views, when you hold a well-reasoned belief that $X$ and an equally informed colleague disagrees with you about whether $X$, you should lower your confidence in $X$ dramatically, or - to state it in terms of categorical beliefs - you should abandon your belief and suspend judgment on the issue.

Let's imagine that you've reasoned your way toward such a view, and that you're the sort of person who acts on the views they hold. Imagine further that you have a well-reasoned belief on some other complex issue, say, you believe that we have libertarian free will. Now, to your dismay, you find yourself in a crossfire: your friend metaphysician Milo thinks that there's no libertarian free will, while your friend epistemologist Evelyn thinks that one should not abandon one's well-reasoned belief when faced with a disagreeing peer. Call this scenario Double Disagreement. The question now is how should you adjust your beliefs. For starters, your conciliatory view appears to self-defeat, or call for abandoning itself. Just instantiate $X$ with it! There's disagreement of the right sort, and so you should abandon your view. And to make the matters worse, there's something in the vicinity of inconsistency around the corner. Think about what are you to do about your belief in free will. Since there's no antecedent reason to start by applying the conciliatory view to itself, you've two lines of argument supporting opposing conclusions: that you should abandon your belief

\footnotetext{
${ }^{1}$ There are a few accounts of epistemic peerhood in the literature. Central to all of them is the idea that your epistemic peer is your epistemic equal. In what follows, I'm going to rely on an intuitive understanding of the notion. It's important to note, though, that epistemic peerhood is always relative to a subject-matter: we can be peers in matters pertaining to social epistemology without being peers in matters pertaining to the philosophy of mathematics. For more on the notion, see, e.g., (Gelfert 2011), (King 2012), (Matheson 2015b, Ch. 2), and (Matheson 2018).

${ }^{2}$ For defenses of conciliatory views see, e.g., (Christensen 2007, 2011, 2016), (Elga 2007), (Feldman 2005, 2006, 2009), and (Matheson 2015b).
} 
in the existence of free will, and that it's not the case that you should. On the one hand, it'd seem that you should drop the belief, in light of your disagreement with Milo and your conciliatory view. On the other, there's the following line of argument too. Your conciliatory view self-defeats, and, once it does, your disagreement with Milo loses its epistemic significance. But if the disagreement isn't significant for you, then it's fine for you to keep your belief in the existence of free will, implying that it's not the case that you should abandon it. ${ }^{3}$

Although this was sketchy and quick, you should agree that the advocates of strong conciliatory views face a challenge: it looks like their views issue inconsistent recommendations in Double Disagreement and other scenarios sharing its structure. What's more, Christensen (2013), Elga (2010), and others have forcefully argued that this challenge generalizes to all types of conciliatory views, whether they be calling for strong, moderate, or even minimal conciliation. ${ }^{4}$ We'll call this challenge the self-defeat objection to conciliatory views. ${ }^{5}$

Advocates of conciliatory views have taken this objection very seriously, offering various ingenious responses to it. Thus, Bogardus (2009) argues that we have a special rational insight into the truth of conciliationism, making it immune from disagreements about it. ${ }^{6}$ Elga proposes modifying conciliationism, with the view of making beliefs in it exempt from its scope of application. Christensen (2013) suggests that cases like Double Disagreement are inherently unfortunate or tragic, and that what they reveal is that conciliatory views can lead to inevitable violations of "epistemic ideals", and not that these views are false.

\footnotetext{
${ }^{3}$ It's worth pointing out that your situation might be even worse. If we suppose, as seems reasonable, that one shouldn't be abandoning one's well-reasoned beliefs willy-nilly, then we can reason to the conclusion that you should abandon your belief in the existence of free will and that you should also keep it.

${ }^{4}$ According to moderate conciliatory views, when you hold a well-reasoned belief that $X$ and find yourself in a disagreement of the right sort, you should lower your confidence in $X$ at least a little. But by how much exactly? Well, typically such views require that, in answering this question, you factor in your own competence in reasoning about $X$-like matters, as well as your colleague's, or, rather, your degree of confidence in these competences. But, then, it's easy enough to imagine scenarios prompting even such more moderate views to self-defeat: just suppose that you find yourself disagreeing over $X$ and that your confidence in your own competence in reasoning about $X$-like matters is extremely low, while your confidence in your colleague's competence in reasoning about $X$-like matters is extremely high. See, e.g., (Christensen 2013, 2021), (Decker 2014), (Elga 2010), and (Littlejohn 2013) for more one this.

${ }^{5}$ This objection is discussed in, e.g., (Christensen 2013), (Decker 2014), (Elga 2010), (Littlejohn 2013, 2020), (Matheson 2015a,b), and (Weatherson 2013). While it isn't the only concern about conciliatory views that stems from cases involving disagreements about the epistemic significance of disagreement, it's the one that strikes me as the most pressing and the one that I'm going to focus on here. The literature discusses (at least) three other concerns: the first is that most actual advocates of conciliatory views aren't rational in holding onto their viewssee, e.g., (Christensen 2021), (Decker 2014), (Kelly 2005), (Littlejohn 2013), and (Matheson $2015 \mathrm{a}, \mathrm{b})$. The second is that a conciliationist has to abandon her view when repeatedly disagreeing over conciliationism with a stubborn opponent-see (Decker 2014), (Elga 2010), and (Weatherson 2013). And the third is that a conciliationist can't maintain any stable view on the correct way to respond to disagreement-see (Christensen 2013) and (Weatherson 2013). The literature appears to have converged on the idea that, from these three concerns, only the first might pose a genuine problem. I say a little more about it in footnote 58 .

${ }^{6}$ See also (Titelbaum 2015) who argues, roughly, that everyone has to have a priori justification for conciliatory views.
} 
Matheson (2015a) invokes "higher-order recommendations" and evidentialism to respond to the objection. ${ }^{7}$ And Pittard (2015) argues that the agent who finds herself in Double Disagreement has no way of "deferring" to her opponent both at the level belief and the level reasoning, and that, therefore, it's rational for her to refuse to abandon her belief in conciliationism. Even without looking at these responses in any more detail, it should be clear that they all either incur intuitive costs or substantially modify conciliationism. ${ }^{8}$ So the advocates of conciliatory views should welcome a less committal and more conservative response to the objection.

One of the two main goals of this paper, then, is to develop such a response. The second one is to present a formal model that captures the core idea behind conciliatory views using the resources from the defeasible logic paradigm. This model, I contend, is particularly useful for exploring the structure of conciliatory reasoning in cases like Double Disagreement and, therefore, also working out a response to the self-defeat objection. In a word, it'll help us see two things. First, the recommendations that conciliatory views issue in such cases aren't, in fact, inconsistent. And second, in those cases where these recommendations may appear incoherent to us - that is, when they say, roughly, that you should abandon your belief in conciliationism and still conciliate in response to the disagreement about free will - they actually call for the correct and perfectly reasonable response. ${ }^{9}$

The remainder of this paper is structured as follows. Section 2 sets up the model and sharpens the objection: Section 2.1 formulates a defeasible reasoner, or a simple logic with a consequence relation at its core; Section 2.2 embeds the core idea behind conciliationism in it; and Section 2.3 turns to cases like Double Disagreement, leading to a formal version of the concern that conciliatory views self-defeat. We then address it in two steps. Section 3 is concerned with a, by and large, technical problem, but, by solving it, we will have shown that conciliatory views do not issue inconsistent recommendations when they turn on themselves. Section 4, in turn, is concerned with explaining why even those recommendations of conciliatory views that may, at first, strike us as incoherent actually call for rational responses. The key role in this is played by the notion of (rational) degrees of confidence. These three sections are followed by a brief conclusion and an appendix, verifying the main observations.

\footnotetext{
${ }^{7}$ See (Matheson 2015a, especially, pp. 153-7) and (Matheson 2015b, Sec. 4). For evidentialism, see (Conee \& Feldman 2004).

${ }^{8} \mathrm{Cf}$. (Christensen 2021). I won't engage with these responses in what follows for reasons of space, but I will provide a classification of responses to the objection in Section 3.4.

${ }^{9}$ Here the model points in the same direction as the two most recent responses to the self-defeat objection, (Christensen 2021) and (Littlejohn 2020). I'll say more about them in Sections 3.4 and 4.3 .
} 


\section{Conciliatory reasoning in default logic}

\subsection{Basic defeasible reasoner}

This section defines a simple defeasible reasoner. The particular reasoner we'll be working with is a form of default logic. ${ }^{10}$ The core idea behind it is to supplement the standard (classical) logic with a special set of default rules representing defeasible generalizations, with a view of being able to derive a stronger set of conclusions from a given set of premises. We assume the language of ordinary propositional logic as our background and represent default rules as pairs of (vertically) ordered formulas: where $X$ and $Y$ are arbitrary propositions, $\frac{X}{Y}$ will stand for the rule that lets us conclude $Y$ from $X$ by default. To take an example, let $B$ be the proposition that Tweety is a bird and $F$ the proposition that Tweety flies. Then $\frac{B}{F}$ says that we can conclude that Tweety flies as soon as we have established that he is a bird. We use the letter $r$ (with subscripts) to denote default rules, and make use of the functions Premise $[\cdot]$ and Conclusion['] to pick out, respectively, the premise and the conclusion of some given rule: if $r=\frac{X}{Y}$, then Premise $[r]=X$ and Conclusion $[r]=Y$. The second function can be applied to sets of rules too: where $\mathcal{S}$ is a set of default rules, Conclusion $[\mathcal{S}]$ picks out the conclusions of all rules in $\mathcal{S}$, or, formally, Conclusion $[\mathcal{S}]=\{$ Conclusion $[r]: r \in \mathcal{S}\}$.

We envision an agent reasoning on the basis of a two-part structure $\langle\mathcal{W}, \mathcal{R}\rangle$ where $\mathcal{W}$ is a set of ordinary propositional formulas - the hard information, or the information that the agent is certain of - and $\mathcal{R}$ is a set of default rules - the rules the agent relies on when reasoning. We call such structures contexts and denote them by the letter $c$ (with subscripts).

Definition 1 (Contexts). A context $c$ is a structure of the form $\langle\mathcal{W}, \mathcal{R}\rangle$, where $\mathcal{W}$ is a set of propositional formulas and $\mathcal{R}$ is a set of default rules.

Let's illustrate the notion using a standard example from the artificial intelligence literature, the Tweety Triangle: in the first step, the reasoning agent learns that Tweety is a bird and concludes that Tweety flies. In the second, it learns that Tweety is a penguin, retracts the previous conclusion, and concludes that Tweety doesn't fly. Since the scenario unfolds in two steps, there are two contexts, $c_{1}=\langle\mathcal{W}, \mathcal{R}\rangle$ and $c_{2}=\left\langle\mathcal{W}^{\prime}, \mathcal{R}\right\rangle$. Let $B$ and $F$ be as before, and let $P$ stand for the proposition that Tweety is a penguin. The hard information $\mathcal{W}$ of $c_{1}$ must include $B$ and $P \supset B$, expressing an instance of the fact that all penguins are birds. The set of rules $\mathcal{R}$ of $c_{1}$ (and $c_{2}$ ), in turn, contains the two rules $r_{1}=\frac{B}{F}$ and $r_{2}=\frac{P}{\neg F}$. The first lets the reasoner infer that Tweety can fly, by default, once it has concluded that Tweety is a bird. The second lets the reasoner infer that Tweety cannot fly, by default, once it has concluded that he is a penguin. Notice that $r_{1}$ and $r_{2}$ can be thought of as instances of two

\footnotetext{
${ }^{10}$ The original formulation of default logic is due to Reiter (1980). My presentation draws on the more user-friendly version of Horty (2012).
} 
sensible, yet defeasible principles for reasoning, namely, that birds usually fly and that penguins usually do not. As for $c_{2}=\left\langle\mathcal{W}^{\prime}, \mathcal{R}\right\rangle$, it is just like $c_{1}$, except for its hard information also contains $P$, saying that Tweety is a penguin.

Now we will specify a procedure determining which formulas follow from any given context. It will rely on an intermediary notion of a proper scenario. Given some context $c=\langle\mathcal{W}, \mathcal{R}\rangle$, any subset of its rules $\mathcal{R}$ counts as a scenario based on $c$, but proper scenarios are going to be special, in the sense that, by design, they will contain all and only those rules from $\mathcal{R}$ of which we'll want to say that they should be applied or that they should be in force. As long as this is kept in mind, the following definition of consequence should make good intuitive sense: ${ }^{11}$

Definition 2 (Consequence). Let $c=\langle\mathcal{W}, \mathcal{R}\rangle$ be a context. Then the statement $X$ follows from $c$, written as $c \sim X$, just in case $\mathcal{W} \cup$ Conclusion $[\mathcal{S}] \vdash X$ for each proper scenario $\mathcal{S}$ based on $c$.

Of course, the notion of a proper scenario still has to be defined. It will emerge as a combination of three conditions on the rules included in them. The first of these captures the intuitive idea that a rule has to come into operation, or that it has to be triggered:

Definition 3 (Triggered rules). Let $c=\langle\mathcal{W}, \mathcal{R}\rangle$ be a context and $\mathcal{S}$ a scenario based on it. Then the default rules from $\mathcal{R}$ that are triggered in $\mathcal{S}$ are those that belong to the set $\operatorname{Triggered}_{\mathcal{W}, \mathcal{R}}(\mathcal{S})=\left\{r \in \mathcal{R}: \mathcal{W} \cup\right.$ Conclusion $\left._{S}\right] \vdash$ Premise $[r]\}$.

Applying this definition to the empty scenario $\varnothing$, against the background of the context $c_{1}$, it's easy to see that Triggered $c_{c_{1}}(\varnothing)=\left\{r_{1}\right\}$ : the hard information $\mathcal{W}=\{B, P \supset B\}$ entails $B$, and $B=$ Premise $\left[r_{1}\right]$.

The need for further conditions on proper scenarios reveals itself once we apply Triggered $(\cdot)$ to any scenario against the background of $c_{2}$. While both rules $r_{1}$ and $r_{2}$ come out triggered in every scenario based on it, the only scenario that seems intuitively correct is $\left\{r_{2}\right\}$. This means that we need to specify a further condition, precluding the addition of $r_{1}$ to $\left\{r_{2}\right\}$. And here's one that does the trick:

Definition 4 (Conflicted rules). Let $c=\langle\mathcal{W}, \mathcal{R}\rangle$ be a context, and $\mathcal{S}$ a scenario based on it. Then the rules from $\mathcal{R}$ that are conflicted in the context of $\mathcal{S}$ are those that belong to the set Conflicted $_{\mathcal{W}, \mathcal{R}}(\mathcal{S})=\{r \in \mathcal{R}: \mathcal{W} \cup$ Conclusion $[\mathcal{S}] \vdash$ $\neg$ Conclusion $[r]\}$.

\footnotetext{
${ }^{11}$ This is one of the two natural ways to define consequence in the present framework. It gives us what's called the skeptical consequence in the literature. Alternatively, we could say that $X$ follows from $c$ just in case $\mathcal{W} \cup$ Conclusion $[\mathcal{S}] \vdash X$ for some proper scenario $\mathcal{S}$ based on $c$. This would give us what's known as the credulous consequence. But nothing important hinges on the choice of the definition here: almost all contexts we're going to discuss will have only one proper scenario based on them, and when there's one proper scenario, the definitions produce the same results. I briefly revisit the alternative definition in footnote 28 .
} 
Notice that we have Conflicted $d_{\mathcal{W}, \mathcal{R}}\left(\left\{r_{2}\right\}\right)=\left\{r_{1}\right\}$, saying that the rule $r_{1}$ is conflicted in the context of the scenario $\left\{r_{2}\right\}$, as desired. Now consider the following preliminary definition for proper scenarios:

Definition 5 (Proper scenarios, first pass). Let $c=\langle\mathcal{W}, \mathcal{R}\rangle$ be a context and $\mathcal{S}$ a scenario based on it. Then $\mathcal{S}$ is a proper scenario based on $c$ just in case

$$
\begin{aligned}
& \mathcal{S}=\left\{r \in \mathcal{R}: \quad r \in \text { Triggered }_{\mathcal{W}, \mathcal{R}}(\mathcal{S}),\right. \\
& \left.r \notin \text { Conflicted }_{\mathcal{W}, \mathcal{R}}(\mathcal{S})\right\} .
\end{aligned}
$$

The definition gives us the correct result when applied to $c_{1}$, since the singleton $\mathcal{S}_{1}=\left\{r_{1}\right\}$ comes out as its only proper scenario. But it falls flat when applied to $c_{2}$, since both $\mathcal{S}_{1}$ and $\mathcal{S}_{2}=\left\{r_{2}\right\}$ qualify as proper. There are multiple ways to resolve this problem formally. The one I adopt here is motivated by the broader goal of having the resources which will let us model conciliatory reasoning.

We introduce a new type of exclusionary rules, letting the reasoner take other rules out of consideration. To be able to formulate such rules, we extend the background language in two ways. First, we introduce rule names: every default rule $r_{X}$ is assigned a unique name $\mathfrak{r}_{X}$-the Fraktur script is used to distinguish rule names from the rules themselves. Second, we introduce a special predicate $\operatorname{Out}(\cdot)$, with a view of forming expressions of the form $\operatorname{Out}\left(\mathfrak{r}_{X}\right)$. The intended meaning of $\operatorname{Out}\left(\mathfrak{r}_{x}\right)$ is that the rule $r_{x}$ is excluded or taken out of consideration. ${ }^{12}$ For concreteness, let $\mathfrak{r}_{1}$ be the name of the familiar rule $r_{1}$. Then $\operatorname{Out}\left(\mathfrak{r}_{1}\right)$ says that $r_{1}$ is excluded.

With names and the new predicate in hand, we can formulate a second negative condition on a rule's inclusion in a proper scenario:

Definition 6 (Excluded rules). Let $c=\langle\mathcal{W}, \mathcal{R}\rangle$ be a context, and $\mathcal{S}$ a scenario based on this context. Then the rules from $\mathcal{R}$ that are excluded in the context of $\mathcal{S}$ are those that belong to the set $\operatorname{Excluded}_{\mathcal{W}, \mathcal{R}}(\mathcal{S})=\{r \in \mathcal{R}$ : $\mathcal{W} \cup$ Conclusion $[\mathcal{S}] \vdash \operatorname{Out}(\mathfrak{r})\}$.

Our full definition of proper scenarios, then, runs thus: ${ }^{13}$

Definition 7 (Proper scenarios). Let $c=\langle\mathcal{W}, \mathcal{R}\rangle$ be a context and $\mathcal{S}$ a scenario based on it. Then $\mathcal{S}$ is a proper scenario based on $c$ just in case

$$
\begin{aligned}
\mathcal{S}=\{r \in \mathcal{R}: \quad & r \in \text { Triggered }_{\mathcal{W}, \mathcal{R}}(\mathcal{S}), \\
& r \notin \text { Conflicted }_{\mathcal{W}, \mathcal{R}}(\mathcal{S}), \\
& \left.r \notin \text { Excluded }_{\mathcal{W}, \mathcal{R}}(\mathcal{S})\right\} .
\end{aligned}
$$

According to this definition, a proper scenario $\mathcal{S}$ contains all and only those rules that are triggered and neither conflicted, nor excluded in it.

\footnotetext{
${ }^{12}$ Compare to (Horty 2012, Sec. 5.2).

${ }^{13}$ It pays noting that this definition ignores a technical problem having to do with aberrant contexts that contain what we might call self-triggering chains of rules. The simplest such context is $\langle\mathcal{W}, \mathcal{R}\rangle$ with $\mathcal{W}=\varnothing$ and $\mathcal{R}=\left\{\frac{A}{A}\right\}$. Nothing important hinges on this though. See (Horty 2012, p. 48f) for a discussion of the problem and his Appendix A.1. for a solution.
} 

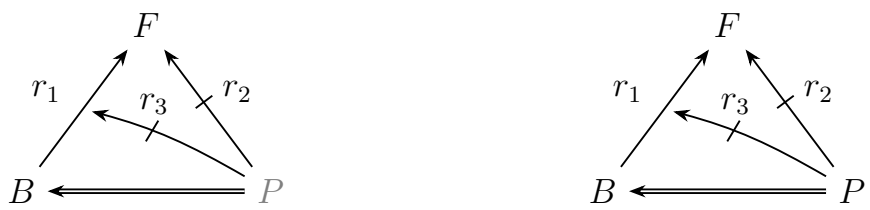

Figure 1: Tweety Triangle, $c_{1}$ (left) and $c_{2}$ (right)

Returning to the example, $c_{1}$ and $c_{2}$ must be supplemented with the rule $r_{3}=\frac{P}{\operatorname{Out}\left(\mathfrak{r}_{1}\right)}$, saying that the rule $r_{1}$ must be taken out of consideration in case Tweety is a penguin. This should make good sense. Since penguins form a peculiar type of birds, it's not reasonable to base one's conclusion that a penguin flies on the idea that birds usually do. It's easy to see that $\mathcal{S}_{1}$ is still the only proper scenario based on $c_{1}$, and that $\mathcal{S}_{3}=\left\{r_{2}, r_{3}\right\}$ is the only proper scenario based on $c_{2}$. So the addition of $r_{3}$ leaves us with a unique proper scenario in each case. ${ }^{14}$ And this gives us the intuitively correct results: given our definition of consequence, the formula $F$, saying that Tweety is able to fly, follows from $c_{1}$, and the formula $\neg F$, saying that Tweety isn't able to fly, follows from $c_{2}$.

With this, our basic defeasible reasoner is complete. We interpret it as a model reasoner: if it outputs $X$ in the context $c$, then it's rational for one to, or one ought to, believe that $X$ in the situation $c$ stands for. And if the reasoner doesn't output $X$ in $c$, then it's not rational for one to, or it's not the case that one ought to, believe that $X$ in the situation $c$ stands for. It may look like the model is committed to the all-or-nothing picture of doxastic attitudes, but it actually can accommodate degree-of-confidence talk as well-more on this in Section 4.

I will sometimes represent contexts as inference graphs. The ones in Figure 1 represent the two contexts capturing the Tweety Triangle. Here's how such graphs should be read: a black node at the bottom of the graph - a node that isn't grayed out, that is - represents an atomic formula from the hard information. A double link from $X$ to $Y$ stands for a proposition of the form $X \supset Y$. A single link from $X$ to $Y$ stands for an ordinary default rule of the form $\frac{\dot{X}}{Y}$, while a crossed out single link from $X$ to $Y$ stands for a default rule of the form $\frac{X}{\neg Y}$. A crossed out link that starts from a node $X$ and points to another link stands for an exclusionary default of the form $\frac{X}{\operatorname{Out}(\mathfrak{r})}$, with the second link representing the rule $r$.

\footnotetext{
${ }^{14}$ This won't hold in general, as there are contexts for which multiple scenarios will qualify as proper.
} 


\subsection{Capturing conciliationism}

Now let's see how the core idea motivating conciliatory views can be captured in the defeasible reasoner. As a first step, consider the following case, in which the conciliatory response seems particularly intuitive:

Mental Math. My friend and I have been going out to dinner for many years. We always tip $20 \%$ and divide the bill equally, and we always do the math in our heads. We're quite accurate, but on those occasions where we've disagreed in the past, we've been right equally often. This evening seems typical, in that I don't feel unusually tired or alert, and neither my friend nor I have had more wine or coffee than usual. I get $\$ 43$ in my mental calculation, and become quite confident of this answer. But then my friend says she got $\$ 45$. I dramatically reduce my confidence that $\$ 43$ is the right answer. ${ }^{15}$

Mental Math describes fairly complex reasoning, and we shouldn't miss three of its features: first, we can distinguish two components in it, the mathematical calculations and the reasoning prompted by the friend's announcement. What's more, it seems perfectly legitimate to call the former the agent's first-order reasoning and the latter her second-order reasoning. Second, the agent's initial confidence in $\$ 43$ being the correct answer is based on her calculations, and it gets reduced because the agent becomes suspicious of them. And third, the friend's announcement provides for a very good reason for the agent to suspect that she may have erred in her calculations.

Bearing this in mind, let's capture the agent's reasoning in the model. To this end, we introduce a new predicate $\operatorname{Seems}(\cdot)$ to our language. Now, $\operatorname{Seems}(X)$ is meant to express the thought that the agent has reasoned to the best of her ability about whether some proposition $X$ is true and come to the conclusion that it is true as a result. I doubt that there's much informative we can say about the reasoning implied in Seems(.). One thing should be clear though: it's going to depend on $X$ and thus also differ from one case to another. If $X$ is a mathematical claim, $\operatorname{Seems}(X)$ implies calculations of the sort described in Mental Math. If $X$ is a philosophical claim, $\operatorname{Seems}(X)$ implies a careful philosophical investigation. Also, note that $\operatorname{Seems}(X)$ is perfectly compatible with $\neg X$. Since the agent is fallible, the fact that she has reasoned to the best of her ability about the issue doesn't guarantee that the conclusion is correct.

Presumably, though, situations where the agent's best reasoning leads her astray are relatively rare, and so it's reasonable for her to go by her best reasoning. After all, she doesn't have any other alternative. This motivates the following default rule schema:

Significance of first-order reasoning: $r(X)=\frac{\operatorname{Seems}(X)}{X}$, or if your best first-order (or domain-specific) reasoning suggests that $X$, conclude $X$ by default.

15 (Christensen 2010, pp. 186-7). 


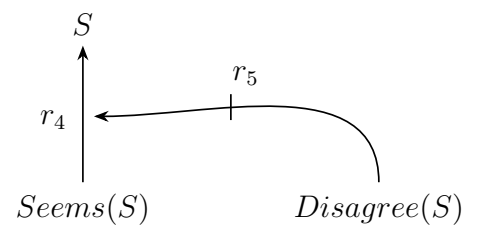

Figure 2: Mental Math, first pass

In Mental Math, we would instantiate the schema with the rule $r_{4}=\frac{\operatorname{Seems}(S)}{S}$, with $S$ standing for the proposition that my share of the bill is $\$ 43$. What the friend's announcement brings into question, then, is exactly the connection between $\operatorname{Seems}(S)$ and $S$. While my mental calculations usually are reliable, now and then I make a mistake. The announcement suggests that this may have happened when I reasoned toward $S$.

To capture the effects of the announcement, we use another designated predicate Disagree $(\cdot)$. The formula Disagree $(X)$ is meant to express the idea that the agent is in genuine disagreement about whether $X$. And I say genuine to distinguish the sorts of disagreements that conciliationists take to be epistemically significant from merely apparent disagreements, such as verbal disagreements and disagreements based on misunderstandings. ${ }^{16}$ So Disagree $(S)$ means that there's a genuine disagreement - between me and my friend - over whether my share of the bill is $\$ 43$. We capture the effects of this disagreement by means of the default rule $r_{5}=\frac{\operatorname{Disagree}(S)}{\operatorname{Out}\left(\mathfrak{r}_{4}\right)}$, which says, roughly, that in case there's genuine disagreement about whether $S$, the rule $r_{4}$, which lets me conclude $S$ on the basis of $\operatorname{Seems}(S)$, is to be excluded. The rule $r_{5}$, then, is what expresses the distinctively conciliatory component of the complex reasoning discussed in Mental Math.

Now notice that $r_{5}$ too instantiates a default rule schema, namely:

Significance of disagreement: $r^{\prime}(X)=\frac{\operatorname{Disagree}(X)}{\operatorname{Out}(\mathfrak{r}(X))}$, or if there's genuine disagreement about whether $X$, stop relying on your first-order reasoning about $X$ by default.

This schema expresses the core idea motivating conciliatory views, or the core of conciliationism, in our model. ${ }^{17}$

\footnotetext{
${ }^{16}$ The distinction is standard-see, for instance, (Matheson 2015b, pp. 7-8).

${ }^{17}$ It's natural to wonder how the core idea behind steadfast views - roughly, the views that say that disagreements aren't epistemically significant - might be captured in the model. My proposal is simple: a model steadfast reasoner would never use the distinctively conciliatory schema $r(X)=\frac{\operatorname{Disagree}(X)}{\operatorname{Out}(\mathfrak{r}(X))}$. Well-known advocates of steadfast views include Kelly (2005, 2010), Titelbaum (2015), and Wedgwood (2010).
} 

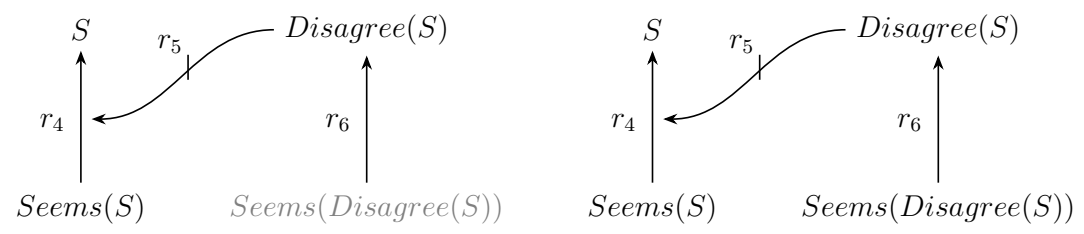

Figure 3: Mental Math, final, $c_{4}$ (left) and $c_{5}$ (right)

As a first pass, we might try to express Mental Math in the context $c_{3}=$ $\langle\mathcal{W}, \mathcal{R}\rangle$ where $\mathcal{W}=\{\operatorname{Seems}(S)$, Disagree $(S)\}$ and $\mathcal{R}=\left\{r_{4}, r_{5}\right\}$ - see Figure 2 for a picture. While $S$ doesn't follow from $c_{3}$, as desired, this context doesn't represent some important features of the scenario. In particular, it misleadingly suggests that the agent doesn't reason to the conclusion that there's genuine disagreement over the bill's amount, but, rather, starts off knowing it for a fact. Admittedly, the description glances over this component of the reasoning, but it's clearly implied by the background story: ".. my friend and I have been going out to dinner for many years.. we've been right equally often.. neither my friend nor I have had more wine or coffee than usual". And nothing stands in the way of capturing the reasoning implied by this description by instantiating the familiar schema $r(X)=\frac{\operatorname{Seems}(X)}{X}$ with $\operatorname{Disagree}(S)$.

We represent Mental Math using the pair of contexts $c_{4}=\langle\mathcal{W}, \mathcal{R}\rangle$ and $c_{5}=\left\langle\mathcal{W}^{\prime}, \mathcal{R}\right\rangle$, with the first standing for the situation before the friend's announcement and the second right after it. The set $\mathcal{W}$ is comprised of the formula Seems $(S)$, and $\mathcal{W}^{\prime}$ of the formulas $\operatorname{Seems}(S)$ and $\operatorname{Seems}(\operatorname{Disagree}(S))$. Both contexts share the same set of rules $\mathcal{R}$, comprised of the familiar rules $r_{4}$ and $r_{5}$, as well as the new rule $r_{6}=\frac{\operatorname{Seems}(\operatorname{Disagree}(S))}{\operatorname{Disagree}(S)}$, another instance of the first-order reasoning schema. The two contexts are depicted in Figure 3. It's not hard to verify that $\left\{r_{4}\right\}$ is the unique proper scenario based on $c_{4}$, and that, therefore, $S$ follows from $c_{4}$, suggesting that, before the announcement, it's rational for the agent to believe that her share of the bill is $\$ 43$. As for $c_{5}$, here again we have only one proper scenario, namely, $\left\{r_{5}, r_{6}\right\}$. This implies that $S$ does not follow from $c_{5}$, suggesting that, after the announcement, it's not rational for the agent to believe that her share of the bill is $\$ 43$. So our model delivers the intuitive result.

It also supports the following take on conciliationism: it's not a simple view, on which you're invariably required to give up your belief in $X$ as soon as you find yourself in disagreement over $X$ with an epistemic peer-or, perhaps, as soon as it's rational for you to think that you're in such a disagreement. Instead, it's a more structured view, saying roughly the following: if your best first-order (or domain-specific) reasoning suggests that $X$ and it's rational for you to believe that you're in disagreement over $X$ with an epistemic peer, then, under normal circumstances, you should bracket your first-order reasoning about $X$ and avoid 
forming beliefs on its basis. ${ }^{18}$

\subsection{Disagreements over disagreement}

Now we can turn to the self-defeat objection. Here's the scenario I used to illustrated it, presented in the first-person perspective:

Double Disagreement. I consider myself an able philosopher with special interests in metaphysics and social epistemology. I've reasoned very carefully about the vexed topic of free will, and I've come to the conclusion that we have libertarian free will. I've also spent a fair amount of time thinking about the issues surrounding peer disagreement, becoming convinced that conciliationism is correct and that one has to give up one's well-reasoned opinion when faced with a disagreeing peer. Then, to my surprise, I discover that my friend metaphysician Milo disagrees with me about the existence of free will, and that my friend epistemologist Evelyn disagrees with me about conciliationism. ${ }^{19}$

Elga (2010) famously argued that cases like this show that conciliatory views are inconsistent. ${ }^{20}$ His line of reasoning runs roughly thus. On the one hand, conciliationism seems to recommend that I abandon my belief in the existence of free will in response to my disagreement with Milo. On the other, conciliationism seems to recommend that I do not abandon my belief in the existence of free will. How? Well, it recommends that I abandon my belief in conciliationism in response to my disagreement with Evelyn. But with this belief gone, my disagreement with Milo seems to lose its epistemic significance for me, implying that it must be okay for me to retain the belief in the existence of free will. Putting the two together, conciliationism appears to support two inconsistent conclusions, that I ought to abandon the belief in free will, and that it's not the case that I ought to.

Now let's see if our model reasoner supports this train of thought. The first step is to capture Double Disagreement in a context, and here already we face a difficulty: we need to model the agent's becoming convinced that conciliationism is correct. We know that conciliationism can be modeled as a specific reasoning policy, but we don't yet know how to model the reasoning that puts such a policy in place. What we'll do, then, is start with a partial representation, and then gradually add the missing pieces. Let $C$ stand for the proposition

\footnotetext{
${ }^{18}$ Notice that the expressions "it's rational for you to believe" and "under normal circumstances" have precise content in the context of the model.

${ }^{19}$ This scenario is a variation on a case discussed by Matheson (2015a), see also (Christensen 2013).

${ }^{20}$ To be precise, Elga doesn't actually discuss a case like Double Disagreement in his paper, but, rather draws an analogy between conciliatory views and the magazine Consumer Reports that reviews products, as well as other consumer ratings magazines, ending up giving inconsistent recommendations: to buy only toaster $X$ and to follow the advice of another magazine, Smart Shopper, that suggest buying only toaster $Y$. The situation with magazines is supposed to be structurally analogous to some case involving a disagreement about conciliationism.
} 

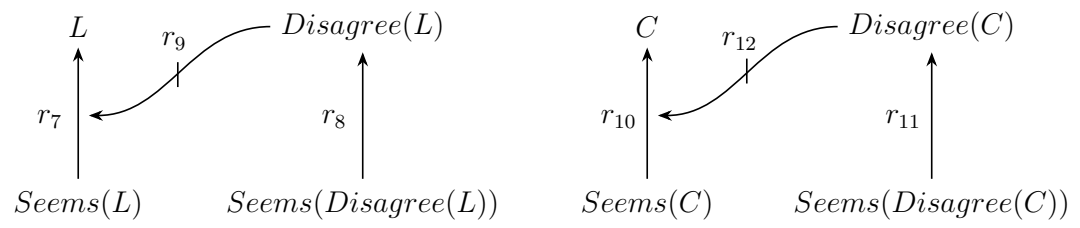

Figure 4: Double Disagreement, first pass

that conciliationism is correct - and think of $C$ as a placeholder to be made precise later on-and $L$ for the proposition that we have libertarian free will. As our first pass, we represent Double Disagreement as the context $c_{6}=\langle\mathcal{W}, \mathcal{R}\rangle$ where $\mathcal{W}$ is comprised of Seems $(L)$, Seems $(C)$, Seems (Disagree $(L))$, and Seems (Disagree $(C)$ ), and where $\mathcal{R}$ contains the following rules:

$r_{7}=\frac{\operatorname{Seems}(L)}{L}:$ If my first-order reasoning about free will suggests that it exists, I conclude that it does indeed exist by default.

$r_{8}=\frac{\operatorname{Seems}(\text { Disagree }(L))}{\text { Disagree }(L)}$ : If my (first-order) reasoning about my disagreement with Milo suggests that it's genuine, I conclude that this disagreement is genuine by default.

$r_{9}=\frac{\text { Disagree }(L)}{\operatorname{Out}\left(\mathfrak{r}_{7}\right)}:$ If there's genuine disagreement over free will, I back off from my first-order reasoning about it by default.

$r_{10}=\frac{\operatorname{Seems}(C)}{C}$ : If my reasoning about the epistemic significance of disagreement suggests that conciliationism is correct, I conclude that it is by default.

$r_{11}=\frac{\text { Seems }(\text { Disagree }(C))}{\text { Disagree }(C)}:$ If my reasoning about my disagreement with Evelyn suggests that it's genuine, I conclude that this disagreement is genuine by default.

$r_{12}=\frac{\text { Disagree }(C)}{\operatorname{Out}\left(\mathfrak{r}_{10}\right)}:$ If there's genuine disagreement over conciliationism, I back off from my first-order reasoning about it by default.

The context $c_{6}$ is depicted in Figure 4.

There's one proper scenario based on $c_{6}$, namely, $\left\{r_{8}, r_{9}, r_{11}, r_{12}\right\}$, and this implies that neither $C$, nor $L$ follow from this context. There's nothing inconsistent here, but the reasoner's response may seem odd: it doesn't draw the conclusion that conciliationism is correct, and yet backs off from its reasoning about free will on distinctively conciliatory grounds. (I'll have much more to 


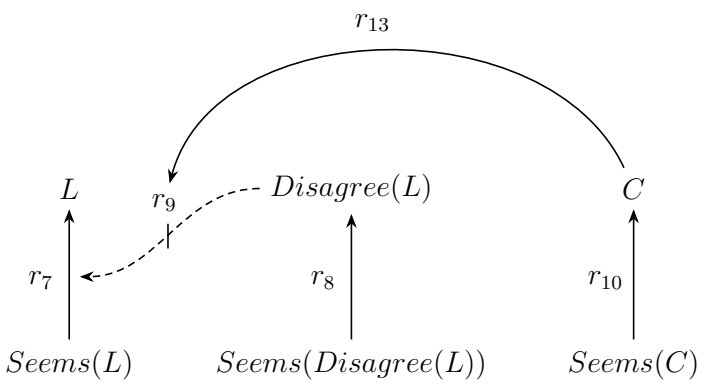

Figure 5: Disagreement with Milo

say about this seeming oddness below.) However, the main problem is that, in $c_{6}$, there's no connection between the proposition saying that conciliationism is correct and the conciliatory reasoning policy, or between $C$ and the rules $r_{9}$ and $r_{12}$.

We're going to put the connection in place, proceeding in two steps and starting by linking $C$ and $r_{9}$. To this end, let's focus on a curtailed version of Double Disagreement in which I never find out that Evelyn disagrees with me over conciliatonism - call this case Disagreement with Milo. We can express it in the context $c_{7}=\langle\mathcal{W}, \mathcal{R}\rangle$, which is like $c_{6}$, except for its hard information $\mathcal{W}$ lacks the formula Seems (Disagree $(C)$ ), and its set of rules lacks $r_{11}$ and $r_{12} \cdot{ }^{21}$

Since $C$ says that conciliationism is correct and $r_{9}$ is an instance of the conciliatory schema $r^{\prime}(X)=\frac{\operatorname{Disagree}(X)}{\operatorname{Out}(\mathfrak{r}(X))}$, it seems reasonable to arrange things in such a way that $C$ is what puts $r_{9}$ in place. To this end, we extend our language with another designated predicate Reasonable $(\cdot)$. Where Out $(\mathfrak{r})$ says that $r$ is taken out of consideration, Reasonable $(\mathfrak{r})$ says that $r$ is a prima facie reasonable rule to follow, or that $r$ is among the rules that the agent could base her conclusions on. The formula Reasonable $\left(\mathfrak{r}_{9}\right)$, in particular, says that $r_{9}$ is a prima facie reasonable rule to follow. ${ }^{22}$ We're going to have to update our defeasible reasoner so that it can take this predicate into account. But first let's connect $C$ and $r_{9}$ by adding the rule $r_{13}=\frac{C}{\text { Reasonable }\left(\mathfrak{r}_{9}\right)}$ to $c_{7}$. The complete context is depicted in Figure 5. Notice that the graph depicting it includes two types of links we haven't seen before. First, there's a single link (standing for $r_{13}$ ) that points to another one and that isn't crossed out. From now on, links of this type will represent rules of the form $\frac{X}{\text { Reasonable }(\mathfrak{r})}$. Second, there's a

\footnotetext{
${ }^{21}$ The rules are left out for the sake of simplicity. Nothing important hinges on this.

${ }^{22}$ It's worth distinguishing the technical notion of reasonable rules that I use here from two senses of reasonable rules implicit in standard default logic and the variant I defined in Section 2.1. My notion is different from the strong sense of reasonable rules in which only those default rules from $\mathcal{R}$ of some context $c=\langle\mathcal{W}, \mathcal{R}\rangle$ that make it into some proper scenario are reasonable. It's also different from the much weaker sense in which all default rules in $\mathcal{R}$ are reasonable.
} 
dashed link (standing for $r_{9}$ ). From now on, links of this type will represent rules that, intuitively, the reasoner can start relying on only after it has concluded that they are prima facie reasonable to follow. ${ }^{23}$

An easy check reveals that Reasonable $\left(\mathfrak{r}_{9}\right)$ and $C$ do, while $L$ does not follow from $c_{7}$. This is the intuitive result, but we can't rest content with what we have here. To see why, consider the context $c_{8}=\left\langle\mathcal{W} \cup\left\{\right.\right.$ Out $\left.\left.\left(\mathfrak{r}_{10}\right)\right\}, \mathcal{R}\right\rangle$ that extends the hard information of $c_{7}$ with the formula $\operatorname{Out}\left(\mathfrak{r}_{10}\right)$, saying that the rule $r_{10}=\frac{\operatorname{Seems}(C)}{C}$ is to be taken out of consideration. It can be verified that the only proper scenario based on $c_{8}$ is $\left\{r_{8}, r_{9}\right\}$, and that, therefore, neither $C$, nor Reasonable $\left(\mathfrak{r}_{9}\right)$, nor $L$ follow from it. But this is the wrong result: the formula $L$ doesn't follow because it gets excluded by the rule $r_{9}$ which was supposed to depend on the reasoner concluding $C$ and Reasonable $\left(\mathfrak{r}_{9}\right)$. Thus, a rule that, intuitively, should have no effects whatsoever ends up precluding the reasoner from deriving $L$.

To make the new predicate and rules like $r_{9}$ do real work, the inner workings of the reasoner have to be modified. And my general strategy here is to let the reasoner use a rule $r$ only on the condition that it can infer a formula of the form Reasonable $(\mathfrak{r})$, just like currently it can use a rule $r$ only on the condition that it can infer its triggering condition, Premise $[r]$. So, from now on, the reasoner is allowed to use a rule $r$ only in case it can infer both Premise $[r]$ and Reasonable(r). And there are two ways for it to infer a formula of the form Reasonable $(\mathfrak{r})$ : from the hard information or by means of other rules. One immediate implication of this is that some Reasonable-formulas are going to have to be included in the hard information. But this should make good sense: the presence of Reasonable $(\mathfrak{r})$ in $\mathcal{W}$ can be understood in terms of the reasoner taking $r$ to be a prima facie reasonable rule to follow from the outset. ${ }^{24}$

In Section 2.1, the central notion of a proper scenario was defined by appealing to three conditions on rules. Now we amend it by adding a fourth one:

Definition 8 (Reasonable rules). Let $c=\langle\mathcal{W}, \mathcal{R}\rangle$ be a context, and $\mathcal{S}$ a scenario based on it. Then the rules from $\mathcal{R}$ that are prima facie reasonable (to follow) in the context of the scenario $\mathcal{S}$ are those that belong to the set Reasonable $_{\mathcal{W}, \mathcal{R}}(\mathcal{S})=\{r \in \mathcal{R}: \mathcal{W} \cup$ Conclusion $[S] \vdash$ Reasonable $(\mathfrak{r})\}$.

Definition 9 (Proper scenarios, revised). Let $\langle\mathcal{W}, \mathcal{R}\rangle$ be a context and $\mathcal{S}$ a

\footnotetext{
${ }^{23}$ More precisely, a rule $r$ will be represented as a dashed link when the corresponding formula Reasonable $(\mathfrak{r})$ can't be derived from the hard information $\mathcal{W}$ of the context. Why aren't all links in the graph from Figure 5 dashed then? Well, it actually doesn't depict $c_{7}$, which is only a preliminary formal rendering of Disagreement with Milo, but rather its final formalization $c_{9}$, which I discuss below. I allow myself to be sloppy in the main text for reasons of exposition.

${ }^{24}$ Here's a way to connect this to standard default logic. In standard default logic, all default rules from the set of rules of a context (default theory) are always prima facie reasonable to follow, in my sense of the term. I generalize this by allowing for rules that are prima facie reasonable to follow from the outset, as well as rules that become prima facie reasonable to follow as a result of applying other rules. Thanks to an anonymous referee for pressing me to clarify the connection.
} 
scenario based on it. Then $\mathcal{S}$ is a proper scenario based on $\langle\mathcal{W}, \mathcal{R}\rangle$ just in case

$$
\begin{aligned}
\mathcal{S}=\{r \in \mathcal{R}: & r \in \text { Reasonable }_{\mathcal{W}, \mathcal{R}}(\mathcal{S}), \\
& r \in \text { Triggered }_{\mathcal{W}, \mathcal{R}}(\mathcal{S}), \\
& r \notin \text { Conflicted }_{\mathcal{W}, \mathcal{R}}(\mathcal{S}), \\
& \left.r \notin \text { Excluded }_{\mathcal{W}, \mathcal{R}}(\mathcal{S})\right\} .
\end{aligned}
$$

With this, we're all done. There's no need to change the definition of consequence. ${ }^{25}$ The next observation shows that our revised reasoner is a conservative generalization of the original one - the proof is provided in the Appendix:

Observation 2.1. Let $c=\langle\mathcal{W}, \mathcal{R}\rangle$ be an arbitrary regular context where no Reasonable-formulas occur - or, more precisely, a context where no subformula of any of the formulas in $\mathcal{W}$ or any of the premises or conclusions of the rules in $\mathcal{R}$ is of the form Reasonable $(\mathfrak{r})$. Then there's a context $c^{\prime}=\langle\mathcal{W} \cup\{\operatorname{Reasonable}(\mathfrak{r})$ : $r \in \mathcal{R}\}, \mathcal{R}\rangle$ that's equivalent to $c$. Or more explicitly, $X$ follows from $c$ if and only if $X$ follows from $c^{\prime}$ for all $X$ such that no subformula of $X$ is of the form Reasonable $(\mathfrak{r})$.

Our final rendering of Disagreement with Milo is the context $c_{9}=\langle\mathcal{W}, \mathcal{R}\rangle$, which is like $c_{7}$, except for its hard information $\mathcal{W}$ also includes the formulas saying that the reasoner takes the rules $r_{7}, r_{8}, r_{10}$, and $r_{13}$ to be prima facie reasonable to follow, that is, $\mathcal{W}$ includes the formulas $\operatorname{Reasonable}\left(\mathfrak{r}_{7}\right)$, Reasonable $\left(\mathfrak{r}_{8}\right)$, Reasonable $\left(\mathfrak{r}_{10}\right)$, and Reasonable $\left(\mathfrak{r}_{13}\right)$. It's not difficult to see that the only proper scenario based on $c_{9}$ is $\left\{r_{8}, r_{9}, r_{10}, r_{13}\right\}$, and so that $C$ does, while $L$ doesn't follow from this context. Thus, our analysis suggests that the correct response to the scenario is to stick to the belief in conciliationism and to abandon the belief in free will. This seems perfectly intuitive. ${ }^{26}$

Now let's zoom in on the other half of the story in Double Disagreement, forgetting about free will for a second and restricting attention to conciliationism. Our preliminary formalization included the formulas $\operatorname{Seems}(C)$ and $\operatorname{Seems}(\operatorname{Disagree}(C))$, as well as the following three rules: $r_{10}=\frac{\operatorname{Seems}(C)}{C}$, $r_{11}=\frac{\operatorname{Seems}(\operatorname{Disagree}(C))}{\operatorname{Disagree}(C)}$, and $r_{12}=\frac{\operatorname{Disagree}(C)}{\operatorname{Out}\left(\mathfrak{r}_{10}\right)}$. We found it wanting because it didn't connect $C$ and $r_{12}$. Now, however, we can complete this formalization by supplementing it with the rule $r_{14}=\frac{C}{\operatorname{Reasonable}\left(\mathfrak{r}_{12}\right)}$, as well

\footnotetext{
${ }^{25}$ An anonymous referee points out that it's not immediately clear how the model set up here might deal with higher-order nestings of reasons, and that it's not clear whether the status of formulas of the form $O u t(r)$ is properly consolidated in the model, given that there are such contexts as $\langle\mathcal{W}, \mathcal{R}\rangle$ where $\mathcal{W}=\{\operatorname{Seems}($ Out $(r))$, Reasonable $(r)\}$ and $\mathcal{R}=\left\{r=\frac{\operatorname{Seems}(\text { Out }(r))}{\operatorname{Out}(r)}\right\}$. The short answer to the second point is that the rule $r$ forms a (degenerate) vicious cycle, and that the tools introduced in Section 3 allow us to deal with cycles of this sort. Unfortunately, I don't have a short answer to the second point, but I'm hoping to provide a detailed answer to it in another paper.

${ }^{26}$ It's only slightly more difficult to see that the only proper scenario based on $c_{8}$, extended with the appropriate Reasonable-formulas, is $\left\{r_{7}, r_{8}\right\}$, and so that $L$ does, while $C$ doesn't follow from it. This, again, seems intuitive.
} 


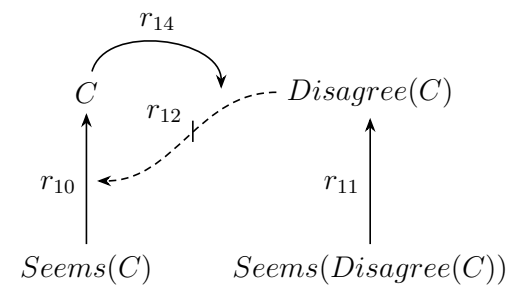

Figure 6: Disagreement with Evelyn

as Reasonable-formulas saying that $r_{10}, r_{11}$, and $r_{14}$ are prima facie reasonable rules. The resulting context $c_{10}$ is depicted in Figure 6. (Note that here and elsewhere formulas of the form Reasonable( $\mathfrak{r}$ ) are not explicitly represented.)

As it turns out, however, there are no proper scenarios based on $c_{10} \cdot{ }^{27}$ This is bad news for the advocates of conciliatory views, since no proper scenarios means that we get $c_{10} \sim X$ for any formula $X$ whatsoever: on our definition of consequence, a formula $X$ follows from a context $c=\langle\mathcal{W}, \mathcal{R}\rangle$ if and only if $\mathcal{W} \cup$ Conclusion $[\mathcal{S}] \vdash X$ for every proper scenario based on $c$. But when there are no proper scenarios, every formula satisfies the right hand side of the biconditional vacuously, and so every formula follows from the context. What's more, the context capturing the entire story recounted in Double Disagreement delivers the same result. Merging $c_{9}$ and $c_{10}$, we acquire the context $c_{11}$, our final representation of the scenario - see Figure 7. Yet again, there are no proper scenarios based on $c_{11}$, and we get $c_{11} \sim X$ for all $X$. Thus, our carefully designed model reasoner seems to suggest that the correct conciliatory response to Double Disagreement is to conclude everything. ${ }^{28}$

We can think of this problem as the formal version of the self-defeat objection to conciliatory views that we started with. In the end, putting forth a model (conciliatory) reasoner which suggests that concluding everything is the correct response to scenarios of a certain shape is much like advancing a (conciliatory)

\footnotetext{
${ }^{27}$ This can be verified by enumeration, going through all subsets of $\mathcal{R}$ one by one. But we can also save ourselves from the tedious exercise by looking only at those scenarios that are antecedently viable. Let's start with three observations. First, if there's a proper scenario at all, it has to include $r_{11}$ : we have $\mathcal{W} \vdash$ Premise $\left[r_{11}\right]$ and there's nothing that might exclude $r_{11}$. Second, a proper scenario will include $r_{14}$ only in case it includes $r_{10}$-otherwise the former wouldn't be triggered. Third, a proper scenario will include $r_{12}$ only in case it includes $r_{11}$ and $r_{14}$-otherwise it either wouldn't be triggered, or it wouldn't be reasonable. There are four scenarios satisfying all of these conditions: $\left\{r_{11}\right\},\left\{r_{10}, r_{11}\right\},\left\{r_{10}, r_{11}, r_{14}\right\}$, and $\left\{r_{10}, r_{11}, r_{12}, r_{14}\right\}$. The first three aren't proper as they fail to include all triggered default rules - e.g., $r_{10}$ is triggered in the context of $\left\{r_{11}\right\}$, but not included in it. And $\left\{r_{10}, r_{11}, r_{12}\right.$, $\left.r_{14}\right\}$ doesn't qualify as proper because $r_{10}$ is excluded in it.

${ }^{28}$ In footnote 11, I mentioned an alternative to our definition of consequence: $X$ follows from $c=\langle\mathcal{W}, \mathcal{R}\rangle$ if and only if $\mathcal{W} \cup\{$ Conclusion $[\mathcal{S}]\}$ for some proper context based on $c$. This definition might look like an improvement-perhaps, in Double Disagreement it's rational to suspend judgment on both $L$ and $C$-and one might hope that switching to the alternative definition would resolve the problem. It would not, however: the alternative definition recommends what we might call universal suspension-even the formulas $\operatorname{Seems}(L)$ and Seems (Disagree $(C))$ don't follow from $c_{10}$.
} 


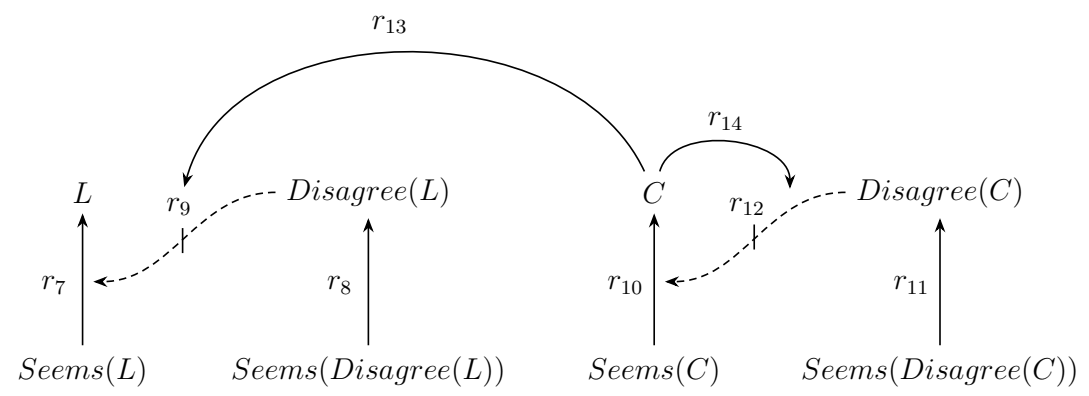

Figure 7: Double Disagreement, final

view that can issue inconsistent recommendations. The fact that this formal problem obtains lends support to Elga's pessimistic conclusion that conciliatory views are inherently flawed.

Luckily, though, the problem can be addressed.

\section{Moving to argumentation theory}

The fact that conciliationism turns on itself in Double Disagreement is only part of the reason why our model reasoner suggests concluding everything. Its other part is something of a technical nuisance: default logic - which our reasoner is based on - isn't particularly well-suited to handle contexts containing what we can call vicious cycles or self-defeating chains of rules. ${ }^{29}$ The main goal of this section is to clear this nuisance. Once it's been cleared, we'll see that conciliatory views do not have to issue inconsistent directives in Double Disagreement and other scenarios like it.

In order to be in a position to handle contexts containing vicious cycles adequately -including the context $c_{11}$ that expresses Double Disagreement and happens to contain such a cycle in the form of the chain of rules $r_{10}-r_{14}-r_{12}$-we go beyond default logic and draw on the resources of a more general formal framework called abstract argumentation theory. As Dung (1995) has shown in his seminal work, default logic, as well as many other formalisms for defeasible reasoning can be seen as special cases of argumentation theory. One implication of this is that it's possible to formulate an argumentation theory-based reasoner that picks out the same consequence relation as our default-logic based reasoner from the previous section. And that's just what we're going to do. For, first, a simple tweak to the new reasoner will let it handle cycles adequately, and, second, we'll need the additional resources of argumentation theory later anyway (to capture degrees of confidence in Section 4).

The remainder of this section is structured as follows. Sections $3.1-3.3$ introduce abstract argumentation, relate it to default logic, and formulate the

\footnotetext{
${ }^{29}$ This problem is well known. See, e.g., (Horty 2012, pp. 59-61) and (Pollock 2009).
} 
more sophisticated reasoner. Section 3.4 returns to Double Disagreement, explains how this reasoner handles it, and contrasts its recommendations with the proposals for responding to the self-defeat objection from the literature.

\subsection{Argument frameworks}

In default logic, conclusions are derived on the basis of contexts. In argumentation theory, they are derived on the basis of argument (or argumentation) frameworks. Formally, such frameworks are pairs of the from $\langle\mathcal{A}, \sim\rangle$, where $\mathcal{A}$ is a set of arguments - the elements of which can be anything - and $\leadsto$ is a defeat relation among them. ${ }^{30}$ Thus, for any two arguments $\mathcal{S}$ and $\mathcal{S}^{\prime}$ in $\mathcal{A}$, the relation $\leadsto$ can tell us whether $\mathcal{S}$ defeats $\mathcal{S}^{\prime}$ or not. ${ }^{31}$ We denote argument frameworks with the letter $\mathcal{F}$. What argumentation theory does is provide a number of sensible ways for selecting the set of winning arguments of any given framework $\mathcal{F}$, the set which, in its turn, determines the conclusions that can be drawn on the basis of $\mathcal{F}$. Since the frameworks we focus on will be constructed from contexts, argumentation theory will let us determine the conclusions that can be drawn on the basis of any given context $c$.

Our logic-based reasoner relies on the notion of a proper scenario to determine the consequences of a context. This notion specifies something like the necessary and sufficient conditions for a rule's counting as admissible or goodthat the rule be reasonable, triggered, not conflicted, and not excluded-and the reasoner can be thought of as selecting such rules in one single step. However, nothing stands in the way of selecting the good rules in a more stepwise fashion. That is, instead of jumping from a context to the scenario containing all and only the admissible rules, we could first select all scenarios whose members satisfy the positive conditions - reasonable and triggered - and later filter out the scenarios whose members do not satisfy the remaining negative conditions - conflicted and excluded. Let's restate the idea, using our formal notation: starting with a context $c=\langle\mathcal{W}, \mathcal{R}\rangle$, in the first step we'd select all and only those scenarios $\mathcal{S} \subseteq \mathcal{R}$ such that, for every $r$ in $\mathcal{S}$,

$$
\mathcal{W} \cup \text { Conclusion }[\mathcal{S}] \vdash \text { Reasonable }(r) \& \operatorname{Premise}[r]
$$

and, in the second step, we'd filter out all of those scenarios $\mathcal{S}$ for which it holds that there's some $r$ in $\mathcal{S}$ such that

$$
\mathcal{W} \cup \text { Conclusion }[\mathcal{S}] \vdash \neg \text { Conclusion }[r] \text { or } \mathcal{W} \cup \text { Conclusion }[\mathcal{S}] \vdash \text { Out }(\mathfrak{r}) \text {. }
$$

After the second step, we'd have access to all and only the good rules. Applying argumentation theory to contexts can be naturally thought of as proceeding in these two steps. The scenarios selected in the first step are the arguments of

\footnotetext{
${ }^{30}$ To forestall a potential misunderstanding, it's worth noting that my use of the term defeat differs from the way it's usually used in argumentation theory, and that it's closer to how, e.g., Dung (1995) and Prakken \& Vreeswijk (2001) use the term attack.

${ }^{31}$ Formally, the defeat relation $\sim$ is a subset of $\mathcal{A} \times \mathcal{A}$. So argument frameworks are directed graphs.
} 


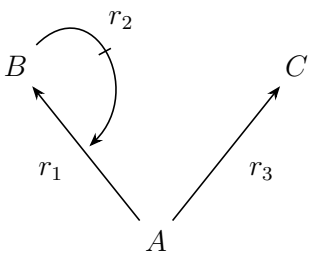

Figure 8: Sample context with a vicious cycle

the argumentation framework based on the given context. And the scenarios that remain standing after the second step are the winning arguments of the framework. The definition of an argument based on a context, then, runs thus:

Definition 10 (Arguments). Let $c=\langle\mathcal{W}, \mathcal{R}\rangle$ be a context and $\mathcal{S}$ a scenario based on it, $\mathcal{S} \subseteq \mathcal{R}$. Then $\mathcal{S}$ is an argument based on $c$ just in case $\mathcal{S} \subseteq$ Triggered $_{\mathcal{W}, \mathcal{R}}(\mathcal{S})$ and $\mathcal{S} \subseteq$ Reasonable $_{\mathcal{W}, \mathcal{R}}(\mathcal{S})$. The set of arguments based on $c$ is the set Arguments $(c)=\{\mathcal{S} \subseteq \mathcal{R}: \mathcal{S}$ is an argument based on $c\}$.

To see the definition at work, let's apply it to a toy case. Consider the context $c_{12}=\langle\mathcal{W}, \mathcal{R}\rangle$ where $\mathcal{W}$ contains $A$, Reasonable $\left(\mathfrak{r}_{1}\right)$, Reasonable $\left(\mathfrak{r}_{2}\right)$, and Reasonable $\left(\mathfrak{r}_{3}\right)$ and $\mathcal{R}$ contains $r_{1}=\frac{A}{B}, \quad r_{2}=\frac{B}{\operatorname{Out}\left(\mathfrak{r}_{1}\right)}$, and $r_{3}=\frac{A}{C}$. The context is depicted in Figure 8. There are eight scenarios based on $c_{12}$, namely, $\mathcal{S}_{0}=\varnothing, \mathcal{S}_{1}=\left\{r_{1}\right\}, \mathcal{S}_{2}=\left\{r_{2}\right\}, \mathcal{S}_{3}=\left\{r_{3}\right\}, \mathcal{S}_{4}=\left\{r_{1}, r_{2}\right\}, \mathcal{S}_{5}=\left\{r_{1}, r_{3}\right\}, \mathcal{S}_{6}=\left\{r_{2}, r_{3}\right\}$, $\mathcal{S}_{7}=\left\{r_{1}, r_{2}, r_{3}\right\}$. Two of these, $\mathcal{S}_{2}$ and $\mathcal{S}_{6}$, fail to qualify as arguments. Each contains a rule that's not triggered in it. Indeed, one glance at the graph depicting $c_{12}$ in Figure 8 is enough to see that $r_{2}$ can't be triggered in any scenario based on $c_{12}$ that doesn't include $r_{1}$. This leaves us with six arguments that comprise the first element $\mathcal{A}$ of the argument framework based on $c_{12}$.

Our next definition specifies the conditions under which one argument defeats another:

Definition 11 (Defeat). Let $c=\langle\mathcal{W}, \mathcal{R}\rangle$ be a context and $\mathcal{S}$ and $\mathcal{S}^{\prime}$ two arguments based on it. Then $\mathcal{S}$ defeats $\mathcal{S}^{\prime}$, written as $\mathcal{S} \leadsto \mathcal{S}^{\prime}$, if and only if there is some rule $r \in \mathcal{S}^{\prime}$ such that either $\mathcal{W} \cup$ Conclusion $[\mathcal{S}] \vdash \neg$ Conclusion $[r]$, or $\mathcal{W} \cup$ Conclusion $[\mathcal{S}] \vdash \operatorname{Out}(\mathfrak{r})$.

Notice how the ideas behind the notions of conflicted and excluded rules get repurposed in this definition. A rule $r$ came out conflicted in the context of a scenario $\mathcal{S}$ just in case $\mathcal{W} \cup$ Conclusion $[\mathcal{S}] \vdash \neg$ Conclusion $[r]$. Now an argument $\mathcal{S}$ defeats another one $\mathcal{S}^{\prime}$ just in case there's a rule $r$ in $\mathcal{S}^{\prime}$ such that $\mathcal{W} \cup$ Conclusion $[\mathcal{S}] \vdash \neg$ Conclusion $[r]$. A similar parallel holds of exclusion. Let's apply the definition to the arguments $\mathcal{S}_{7}=\left\{r_{1}=\frac{A}{B}, r_{2}=\frac{B}{\operatorname{Out}\left(\mathfrak{r}_{1}\right)}, r_{3}=\frac{A}{C}\right\}$ and $\mathcal{S}_{1}=\left\{r_{1}=\frac{A}{B}\right\}$. Since Conclusion $\left[\mathcal{S}_{7}\right]$ entails $\operatorname{Out}\left(\mathfrak{r}_{1}\right)$ and $r_{1}$ is in $\mathcal{S}_{1}$, 


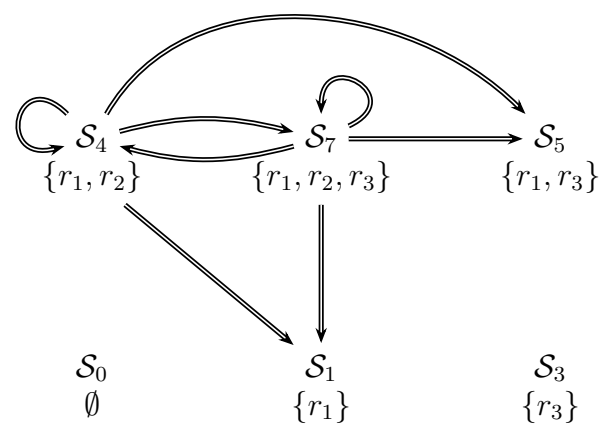

Figure 9: Argument framework based on the sample context $c_{12}$

the argument $\mathcal{S}_{7}$ defeats $\mathcal{S}_{1}$. What's more, given that $r_{1}$ is an element of $\mathcal{S}_{7}$, this argument also self-defeats.

Now we have all that's needed to specify how to construct argument frameworks from contexts:

Definition 12 (Argument frameworks based on contexts). Let $c=\langle\mathcal{W}, \mathcal{R}\rangle$ be an epistemic context. Then the argument framework $\mathcal{F}(c)$ based on $c$ is the pair $\langle\mathcal{A}, \leadsto\rangle$ where $\mathcal{A}=\operatorname{Arguments}(c)$ and $\leadsto$ is the set $\left\{\left(\mathcal{S}, \mathcal{S}^{\prime}\right) \in \mathcal{A} \times \mathcal{A}\right.$ : $\mathcal{S}$ defeats $\left.\mathcal{S}^{\prime}\right\}$.

Figure 9 represents the argument framework $\mathcal{F}\left(c_{12}\right)$ constructed from $c_{12}$. Here's how it should be read: the nodes of the graph represent the arguments in $\mathcal{A}$, and the double arrows between the nodes stand for the defeat relations between arguments. A node with an arrow pointing to itself means that the argument it represent self-defeats.

It'll be useful to introduce some shorthand notation: let $\mathcal{F}=\langle\mathcal{A}, \leadsto\rangle$ be an arbitrary argument framework and $\Gamma$ and $\Gamma^{\prime}$ two sets of arguments from $\mathcal{A}$. When there's an argument $\mathcal{S}$ in $\Gamma$ that defeats some argument $\mathcal{S}^{\prime}$ from $\mathcal{A}$, we write $\Gamma \leadsto \mathcal{S}^{\prime}$; and when there's a pair of arguments $\mathcal{S}$ and $\mathcal{S}^{\prime}$ such that $\mathcal{S}$ is in $\Gamma, \mathcal{S}^{\prime}$ is in $\Gamma^{\prime}$, and $\mathcal{S} \leadsto \mathcal{S}^{\prime}$, we write $\Gamma \leadsto \Gamma^{\prime}$. As an illustration, in the case of $\mathcal{F}\left(c_{12}\right)$, we have $\left\{\mathcal{S}_{0}, \mathcal{S}_{7}\right\} \leadsto \mathcal{S}_{5}$, while we do not have $\left\{\mathcal{S}_{0}, \mathcal{S}_{7}\right\} \leadsto \mathcal{S}_{3}$; and we have $\left\{\mathcal{S}_{0}, \mathcal{S}_{7}\right\} \leadsto\left\{\mathcal{S}_{1}, \mathcal{S}_{3}, \mathcal{S}_{4}\right\}$, while we do not have $\left\{\mathcal{S}_{0}, \mathcal{S}_{7}\right\} \leadsto\left\{\mathcal{S}_{3}\right\}$. Further, when there's no argument $\mathcal{S}$ in $\Gamma$ such that $\mathcal{S}$ defeats $\mathcal{S}^{\prime}$, we write $\Gamma \rtimes \mathcal{S}^{\prime}$; and when there's no pair of arguments $\mathcal{S}$ and $\mathcal{S}^{\prime}$ such that $\mathcal{S}$ is in $\Gamma, \mathcal{S}^{\prime}$ is in $\Gamma^{\prime}$, and $\mathcal{S} \leadsto \mathcal{S}^{\prime}$, we write $\Gamma \rightsquigarrow \Gamma^{\prime}$.

\subsection{Selecting winning arguments}

Now we can turn to argumentation theory proper. As flagged above, the winning argument set of a framework is selected solely on the basis of defeat relations 
between its arguments. ${ }^{32}$ In the literature, the collection of definitions letting one select such sets is called admissibility semantics. You may find it helpful to think of this semantics as serving a function that's similar to the one served by the notion of a proper scenario in the context of default logic. There's one important difference, however. Where default logic didn't offer any choice, the admissibility semantics provides a number of different sensible ways of selecting winning arguments. We're going to focus on two such ways here, beginning with what's called stability semantics:

Definition 13 (Stability semantics). Let $\mathcal{F}=\langle\mathcal{A}, \leadsto\rangle$ be an argument framework and $\Gamma$ a set of arguments from $\mathcal{A}$. Then:

(i) $\Gamma$ is conflict-free if and only if there are no two arguments $\mathcal{S}, \mathcal{S}^{\prime}$ in $\Gamma$ such that $\mathcal{S} \leadsto \mathcal{S}^{\prime}$,

(ii) $\Gamma$ is stable, or a stable extension of $\mathcal{F}$, if and only if

(1) $\Gamma$ is conflict-free, and

(2) $\Gamma$ defeats all the arguments that are not in it, that is, for all $\mathcal{S} \in \mathcal{A} \backslash \Gamma$, $\Gamma \leadsto \mathcal{S}$.

Stability semantics is closely related to default logic, and we'll state the precise connection between the two in a moment. For now, simply note that there are no stable argument sets based on the framework $\mathcal{F}\left(c_{12}\right)$, just like there are no proper scenarios based on the context $c_{12} .{ }^{33}$ The argument sets that are conflictfree, such as $\left\{\mathcal{S}_{0}, \mathcal{S}_{3}\right\}$, fail to defeat all of the arguments that are not in them, and the argument sets that defeat all of the arguments that are not in them, such as $\left\{\mathcal{S}_{0}, \mathcal{S}_{3}, \mathcal{S}_{4}\right\}$, fail to be conflict-free. This is due to the self-defeating chain $r_{1}-r_{2}$.

The alternative to stability semantics we'll use is called preference semantics.

Definition 14 (Preference semantics). Let $\mathcal{F}=\langle\mathcal{A}, \leadsto\rangle$ be an argument framework and $\Gamma$ a set of arguments from $\mathcal{A}$. Then:

(i) $\Gamma$ is conflict-free if and only if there are no $\operatorname{arguments} \mathcal{S}, \mathcal{S}^{\prime}$ in $\Gamma$ such that $\mathcal{S} \leadsto \mathcal{S}^{\prime}$.

(ii) An argument $\mathcal{S}$ in $\mathcal{A}$ is defended by $\Gamma$ if and only if, for all $\mathcal{S}^{\prime}$ (with $\mathcal{S}^{\prime}$ in $\mathcal{A} \backslash \Gamma$ ) such that $\mathcal{S}^{\prime} \leadsto \mathcal{S}$, we have $\Gamma \leadsto \mathcal{S}^{\prime}$.

(iii) $\Gamma$ is a complete extension of $\mathcal{F}$ if and only if

(1) $\Gamma$ is conflict-free, and

\footnotetext{
${ }^{32}$ In general, a framework can have multiple winning sets. This detail is generally important, but not for our purposes.

${ }^{33}$ The latter fact can be verified by enumeration. The intuitively sensible scenario $\mathcal{S}_{3}=\left\{r_{3}\right\}$ doesn't qualify as proper because it does not contain the rule $r_{1}$ which is reasonable, triggered, and neither conflicted, nor excluded in its context.
} 
(2) $\Gamma$ contains all of the arguments it defends.

(iv) $\Gamma$ is preferred, or a preferred extension of $\mathcal{F}$, if and only if $\Gamma$ is a maximal complete extension of $\mathcal{F}$, that is, if and only if there's no other complete extension $\Gamma^{\prime}$ of $\mathcal{F}$ such that $\Gamma \subset \Gamma^{\prime}$.

Let's apply this definition to $\mathcal{F}\left(c_{12}\right)$. The largest conflict-free set of arguments is $\left\{\mathcal{S}_{0}, \mathcal{S}_{1}, \mathcal{S}_{3}, \mathcal{S}_{5}\right\}$. However, it does not defend all of its members: both $\mathcal{S}_{4}$ and $\mathcal{S}_{7}$ defeat $\mathcal{S}_{5}$, and there's nothing in the set that defeats either of them. There are a few sets that are both conflict-free and defend all of their members, namely, $\varnothing,\left\{\mathcal{S}_{0}\right\},\left\{\mathcal{S}_{3}\right\}$, and $\left\{\mathcal{S}_{0}, \mathcal{S}_{3}\right\}$. However, only one of them qualifies as a complete extension, namely, $\left\{\mathcal{S}_{0}, \mathcal{S}_{3}\right\}$. Why do the other sets fail to qualify? Well, take $\left\{\mathcal{S}_{0}\right\}$ as example. A complete set is supposed to contain all the arguments it defends, and a minute's reflection reveals that there's an argument that $\left\{\mathcal{S}_{0}\right\}$ defends that's not in it, namely, $\mathcal{S}_{3}$. What's more, the set $\left\{\mathcal{S}_{0}, \mathcal{S}_{3}\right\}$ also happens to be the unique preferred extension of $\mathcal{F}\left(c_{12}\right)$.

Preference semantics has a clear intuitive rationale. Any conflict-free set of arguments that defends itself - that is, any complete extension - is a desirable state to occupy: it is consistent, and it has a rejoinder to every attack on it. And there's a clear sense in which a preferred set of arguments is an even more desirable state: it's still consistent, it still has a rejoinder to every attack on it, and it's also as big of an argument set of this sort as there can be. So if we're looking to select winning argument sets, preferred extensions are very natural candidates. ${ }^{34}$

While we have labeled various kinds of argument sets, we haven't stated the conditions under which a formula follows from such a set. The following definition rectifies the omission:

Definition 15. Where $\mathcal{F}(c)$ is an argument framework constructed from some context $c=\langle\mathcal{W}, \mathcal{R}\rangle$ and $\Gamma$ is a set of arguments based on $\mathcal{F}(c)$, a statement $X$ is a conclusion of $\Gamma$ if and only if there is some argument $\mathcal{S}$ in $\Gamma$ such that $\mathcal{W} \cup$ Conclusion $[\mathcal{S}] \vdash X$.

\footnotetext{
${ }^{34}$ It pays noting that argumentation theory offers alternative ways of selecting winning sets of arguments too. The one that's at least as important as stability and preference semantics is called grounded semantics. It selects the so-called grounded extension, or the (set theoretically) minimal complete extensions of argument frameworks. Just as it is in the case of preferred extensions - but not the stable ones - grounded extensions are guaranteed to exist, whether or not the underlying argument frameworks contains self-defeating arguments. Given that our goal here is to formulate a defeasible reasoner that draws sensible conclusions in the presence of self-defeating chains of rules, nothing of importance would change were we to adopt grounded semantics instead of preference semantics: for most, if not all, of the contexts and argument frameworks based on them that are discussed in this paper, grounded and preferred extensions coincide. One reason that speaks against the grounded semantics, however, is that it seems to mishandle cases that have the shape of the famous Nixon Diamond. Consider the context $\langle\mathcal{W}, \mathcal{R}\rangle$ where $\mathcal{W}=\{\neg(A \& B)\}$ and $\mathcal{R}=\left\{\frac{\mathrm{T}}{A}, \frac{\mathrm{T}}{B}\right\}$. Arguably, the formula $A \vee B$ should follow from this context, and it does, if we use the preference semantics. But if we use the grounded semantics, it does not. Thanks to an anonymous referee for the suggestion to find a tie-breaker between the two semantics.
} 
With this, we have all the elements we need to define two distinct consequence relations. Both specify when a formula $X$ follows from a context $c$. Both take a circuitous route, utilizing the resources of argumentation theory. The only difference between them is that the first relies on stability and the second on preference semantics:

Definition 16 (Consequence, stable). Let $c=\langle\mathcal{W}, \mathcal{R}\rangle$ be a context. Then the statement $X$ follows from $c$ according to stability semantics, written as $c r_{s} X$, just in case it is a conclusion of every stable extension of the argument framework $\mathcal{F}(c)$.

Definition 17 (Consequence, preferred). Let $c=\langle\mathcal{W}, \mathcal{R}\rangle$ be a context. Then the statement $X$ follows from $c$ according to preference semantics, written as $c \sim_{p} X$, just in case it is a conclusion of every preferred extension of the argument framework $\mathcal{F}(c)$.

The promised connection between default logic and stability semantics can now be stated in the form of an observation - the proof of which is provided in the Appendix:

Observation 3.1. Let $c=\langle\mathcal{W}, \mathcal{R}\rangle$ be a context and $X$ an arbitrary formula. Then $X$ follows from $c$ in default logic, $c \sim X$, if and only if $X$ follows from $c$ according to stability semantics, $c r_{s} X$.

So stability semantics expresses default logic in argumentation-theoretic terms, inheriting its virtues and vices. We have seen one of its vices: default logic collapses in the presence of self-defeating chains. ${ }^{35}$

My proposal is that we switch from the consequence relation picked out by both default logics and stability semantics to the one picked out by the preference semantics. The move is not ad hoc, because there is a clear sense in which preference semantics is a conservative generalization of stability semantics, and, thus, also of default logic. This sense is captured by the following observation - its proof is, again, given in the Appendix:

Observation 3.2. Let $c=\langle\mathcal{W}, \mathcal{R}\rangle$ be a context and $\mathcal{F}(c)=\langle\mathcal{A}, \leadsto\rangle$ an argument framework constructed from it. If $\mathcal{F}(c)$ does not contain either odd cycles of defeat or infinite chains of defeat, then $c \sim_{s} X$ if and only if $c r_{p} X$.

\footnotetext{
${ }^{35}$ Interestingly, argumentation theory provides an explanation of why this happens. First, notice that any argument containing a self-defeating chain of rules self-defeats. Second, recall that stable extensions are conflict-free argument sets that defeat all the arguments that aren't in them. And now suppose that we have some framework $\mathcal{F}=\langle\mathcal{A}, \leadsto\rangle$, that $\mathcal{S}$ is some selfdefeating argument from $\mathcal{A}$, and that $\Gamma$ is a would-be stable extension of $\mathcal{F}$. How would $\Gamma$ relate to $\mathcal{S}$ ? Clearly, $\Gamma$ can't include $\mathcal{S}$. Otherwise, it wouldn't be conflict-free. So $\Gamma$ must defeat $\mathcal{S}$. But, as long as $\mathcal{F}$ isn't based on a very peculiar sort of context, there's just not going to be an independent argument in $\Gamma$ - that is, an argument that's neither a subset, nor a superset of $\mathcal{S}$ - that would defeat $\mathcal{S}$. And if there's no such argument, $\Gamma$ can't qualify as a stable extension. The demanding character of stability semantics becomes especially clear when we contrast it with preference semantics. Where the latter requires that a winning argument set has a rejoinder to every attack on it, the former requires that a winning set attacks every argument that's not in it.
} 

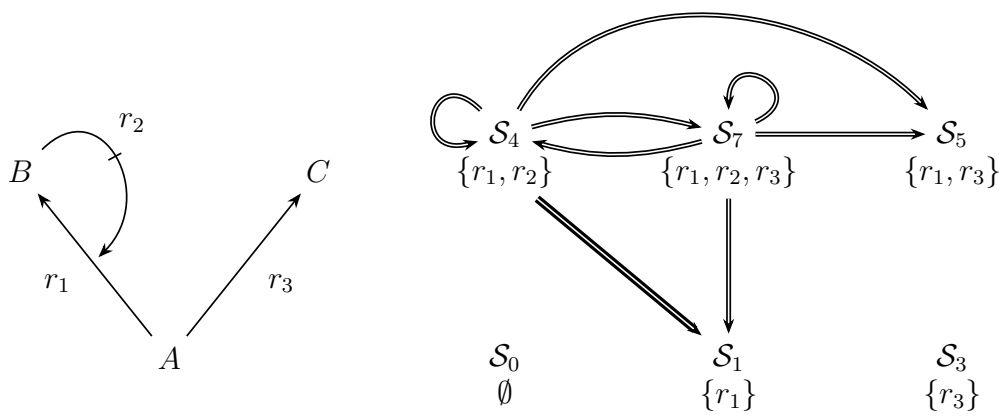

Figure 10: Sample context $c_{12}$ and the argumentation framework $\mathcal{F}\left(c_{12}\right)$, again

From this, it immediately follows that preference semantics gives the same results as default logic in all contexts that do not contain any self-defeating chains of rules. ${ }^{36}$ If a given context contains such a chain, default logic returns the trivial set, while preference semantics returns more meaningful consequences. The toy context $c_{12}$ is a case in point. When we run default logic on it, we get $c_{12} \sim X$ for any formula $X$ whatsoever, while, when we rely on the preference semantics, we get the more reasonable $c_{12} \quad{ }_{p} C$ and $c_{12} \psi_{p} B$. Here and in general, preference semantics effectively disregards self-defeating chains of rules and draws conclusions on the basis of those rules only that are independent of such chains.

\subsection{Minimal arguments and basic defeat}

Before we return to Double Disagreement, it'll be useful to introduce an alternative way of thinking about argumentation frameworks that makes analyzing complex frameworks easier.

If we take another look at the framework $\mathcal{F}\left(c_{12}\right)$-depicted alongside the context $c_{12}$ it's constructed from in Figure 10-we should realize that there's an intuitive sense in which some of its arguments are basic and others are not. While $\mathcal{S}_{1}=\left\{r_{1}\right\}, \mathcal{S}_{3}=\left\{r_{3}\right\}, \mathcal{S}_{4}=\left\{r_{1}, r_{2}\right\}, \mathcal{S}_{5}=\left\{r_{1}, r_{3}\right\}$, and $\mathcal{S}_{7}=\left\{r_{1}, r_{2}, r_{3}\right\}$ all qualify as arguments based on $c_{12}$, there seems to be a qualitative difference between the first three, $\mathcal{S}_{1}, \mathcal{S}_{3}$, and $\mathcal{S}_{4}$, on the one hand, and $\mathcal{S}_{5}$ and $\mathcal{S}_{7}$, on the other. First off, $\mathcal{S}_{1}, \mathcal{S}_{3}$, and $\mathcal{S}_{4}$ are the (set-theoretically) smallest arguments allowing us to derive, respectively, $B, \operatorname{Out}\left(\mathfrak{r}_{1}\right)$, and $C$. Thus, while we have both $\mathcal{W} \cup$ Conclusion $\left[\mathcal{S}_{1}\right] \vdash B$ and $\mathcal{W} \cup$ Conclusion $\left[\mathcal{S}_{7}\right] \vdash B$, only in the case of $\mathcal{S}_{1}$ can we say that there's no smaller argument that would let us derive $B$. And second, $\mathcal{S}_{5}$ and $\mathcal{S}_{7}$ are naturally thought of as aggregates of the basic arguments:

\footnotetext{
${ }^{36}$ The presence of a self-defeating chain of rules in $c$ typically means that the argument framework $\mathcal{F}(c)$ constructed from $c$ contains at least one self-defeating argument - this doesn't happen only in those cases where not a single arguments of $\mathcal{F}(c)$ subsumes the chain. But self-defeating argument are odd cycles of defeat. So if $\mathcal{F}(c)$ has no odd cycles of defeat, then $c$ cannot contain a self-defeating chains of rules.
} 
$\mathcal{S}_{5}$ combines $\mathcal{S}_{1}$ and $\mathcal{S}_{3}$, while $\mathcal{S}_{7}$ combines $\mathcal{S}_{1}, \mathcal{S}_{3}$, and $\mathcal{S}_{4}$. What's more, we can identify a basic defeat relation between arguments. There's, again, an intuitive sense in which the real action happens between the smallest argument supporting the conclusion $\operatorname{Out}\left(\mathfrak{r}_{1}\right)$, namely, $\mathcal{S}_{4}$, and the smallest argument containing $r_{1}$, namely, $\mathcal{S}_{1}$. (In Figure 10, this relation is represented by the highlighted arrow.) The defeat relations between the other arguments depend on it in a way we can make precise: for any two arguments $\mathcal{S}, \mathcal{S}^{\prime}$ in $\mathcal{F}\left(c_{12}\right)$, we have $\mathcal{S} \leadsto \mathcal{S}^{\prime}$ only if the basic argument $\mathcal{S}_{4}$ is a part of $\mathcal{S}, \mathcal{S}_{4} \subseteq \mathcal{S}$, and the basic argument $\mathcal{S}_{1}$ is a part of $\mathcal{S}^{\prime}, \mathcal{S}_{1} \subseteq \mathcal{S}^{\prime}$.

We're going to capture these intuitive ideas of basic arguments and defeat in a mathematically precise way, and then show how they let us define an alternative relation of defeat between the arguments based on some context $c$ that's extensionally equivalent to the one we're already familiar with.

The first step in specifying this relation is to select those arguments from Arguments(c) that seem basic. Notice here that an argument that's basic with respect to one rule or one formula doesn't have to count as basic with respect to another rule or formula. Consider our example again. The scenario $\mathcal{S}_{4}=$ $\left\{r_{1}, r_{2}\right\}$ seems basic with respect to both the rule $r_{2}$ and the formula Out $\left(\mathfrak{r}_{1}\right)$, since there's no smaller argument that would either contain $r_{2}$, or let us derive $\operatorname{Out}\left(\mathfrak{r}_{1}\right)$. However, $\mathcal{S}_{4}$ is not basic with respect to $r_{1}$ and the formula $B$, since there's the smaller $\mathcal{S}_{1}=\left\{r_{1}\right\}$ which contains $r_{1}$ and let's us derive $B$. What this means is that the formal notion capturing the intuitive idea of basicness must be relativized, to a rule or a formula. But otherwise, the basic, or minimal, arguments just are the (set-theoretically) smallest arguments we can find:

Definition 18 (Minimal arguments, with respect to rules). Let $c=\langle\mathcal{W}, \mathcal{R}\rangle$ be a context and $r$ a rule from $\mathcal{R}$. Then the $r$-minimal arguments, in the context of $c$, are those arguments that belong to the set

$$
\begin{aligned}
\operatorname{Minimal}_{\mathcal{F}(c)}(r)=\{\mathcal{S} \in \operatorname{Arguments}(c): & r \in \mathcal{S} \text { and } \\
& \nexists \mathcal{S}^{\prime} \in \operatorname{Arguments}(c) \text { such that } \\
& (1) r \in \mathcal{S}^{\prime} \text { and } \\
& \left.(2) \mathcal{S}^{\prime} \subset \mathcal{S}\right\} .
\end{aligned}
$$

Definition 19 (Minimal arguments, with respect to formulas). Let $c=\langle\mathcal{W}, \mathcal{R}\rangle$ be a context and $X$ a formula of our language. Then the $X$-minimal arguments, in the context of $c$, are those arguments that belong to the set

$$
\begin{aligned}
\operatorname{Minimal}_{\mathcal{F}(c)}(X)=\{\mathcal{S} \in \operatorname{Arguments}(c): & \mathcal{W} \cup \operatorname{Conclusion}[\mathcal{S}] \vdash X \text { and } \\
& \nexists \mathcal{S}^{\prime} \in \operatorname{Arguments}(c) \text { such that } \\
& (1) \mathcal{W} \cup \text { Conclusion }\left[\mathcal{S}^{\prime}\right] \vdash X, \\
& \left.(2) \mathcal{S}^{\prime} \subset \mathcal{S}\right\} .
\end{aligned}
$$

The plural in the definitions isn't accidental. In general, there can be multiple $r$ - or $X$-minimal arguments, as our next example makes plain. Consider 


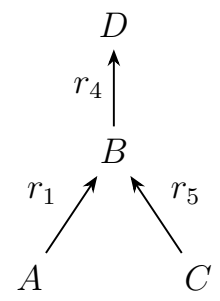

Figure 11: Multiple basic arguments

the context $c_{13}=\langle\mathcal{W}, \mathcal{R}\rangle$ with $\mathcal{W}=\left\{A, C\right.$, Reasonable $\left(\mathfrak{r}_{1}\right)$, Reasonable $\left(\mathfrak{r}_{4}\right)$, Reasonable $\left.\left(\mathfrak{r}_{5}\right)\right\}$ and $\mathcal{R}$ consisting of the rules $r_{1}=\frac{A}{B}, \quad r_{4}=\frac{B}{D}$, and $r_{5}=\frac{C}{B}$. A glance at the inference graph depicting this context (see Figure 11) is enough to realize that there are two alternative ways of reaching $D$, by means of the chain $r_{1}-r_{4}$ and by means of the chain $r_{5}-r_{4}$. And, indeed, if we apply Definition 19 to $c_{13}$, two arguments come out as $D$-minimal, namely, $\left\{r_{1}, r_{4}\right\}$ and $\left\{r_{4}, r_{5}\right\}$.

Now let's turn to basic defeat. Our next definition might look somewhat involved, but all it does is capture the intuition we started with. For two arguments $\mathcal{S}$ and $\mathcal{S}^{\prime}$ to stand in the relation of basic defeat, there has to be a rule $r$ such that $\mathcal{S}^{\prime}$ is $r$-minimal and $\mathcal{S}$ is either $\neg$ Conclusion $[r]$ - or $O u t(\mathfrak{r})$-minimal.

Definition 20 (Basic defeat). Let $c=\langle\mathcal{W}, \mathcal{R}\rangle$ be a context and $\mathcal{S}$ and $\mathcal{S}^{\prime}$ two arguments of the framework $\mathcal{F}(c)$. Then $\mathcal{S}$ stands in the relation of basic defeat to $\mathcal{S}^{\prime}$, written as $\mathcal{S} \sim_{b} \mathcal{S}^{\prime}$, if and only if there is some rule $r \in \mathcal{R}$ such that

(i) $\mathcal{S}^{\prime}$ is in $\operatorname{Minimal}_{\mathcal{F}(c)}(r)$ and

(ii) either (1) or (2):

(1) $\mathcal{W} \cup$ Conclusion $[\mathcal{S}] \vdash \neg$ Conclusion $[r]$ and $\mathcal{S}$ is in $\operatorname{Minimal}_{\mathcal{F}(c)}(\neg$ Conclusion $[r])$,

(2) $\mathcal{W} \cup$ Conclsuion $[\mathcal{S}] \vdash \operatorname{Out}(\mathfrak{r})$ and $S$ is in $\operatorname{Minimal}_{\mathcal{F}(c)}(\operatorname{Out}(\mathfrak{r}))$.

Returning to $c_{12}$, it can be verified that the only two arguments that stand in the basic defeat relation are $\mathcal{S}_{4}$ and $\mathcal{S}_{1}$. For $\mathcal{S}_{4}$ is the only element of the set $\operatorname{Minimal}_{\mathcal{F}\left(c_{12}\right)}\left(\operatorname{Out}\left(\mathfrak{r}_{1}\right)\right)$ and $\mathcal{S}_{1}$ is the only element of $\operatorname{Minimal}_{\mathcal{F}\left(c_{12}\right)}\left(r_{1}\right)$. And given that Conclusion $\left[\mathcal{S}_{4}\right]$ entails $\operatorname{Out}\left(\mathfrak{r}_{1}\right)$, we have $\mathcal{S}_{4} \gg_{b} \mathcal{S}_{1}$.

The relation of basic defeat can then be extrapolated to arguments of arbitrary complexity, as follows:

Definition 21 (Defeat, alternative definition). Let $c=\langle\mathcal{W}, \mathcal{R}\rangle$ be a context and $\mathcal{S}$ and $\mathcal{S}^{\prime}$ two arguments based on it. Then $\mathcal{S}$ defeats $\mathcal{S}^{\prime}$, written as $\mathcal{S} \sim_{a} \mathcal{S}^{\prime}$, if 


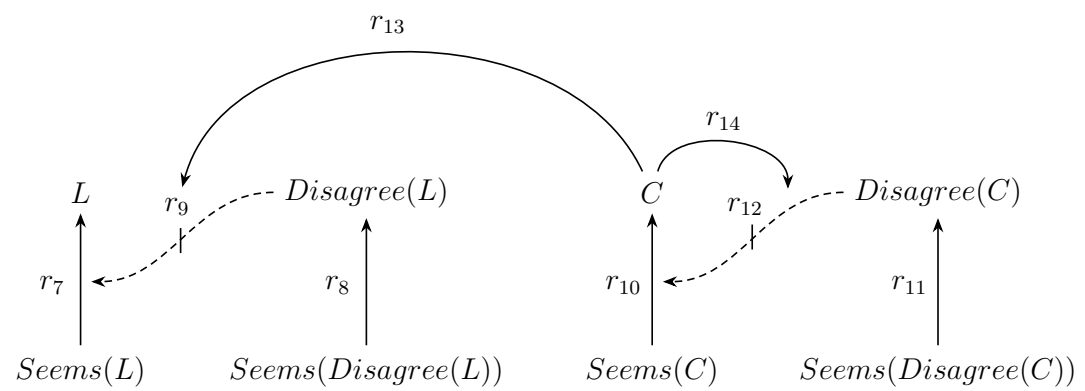

Figure 12: Double Disagreement, again

and only if there is an $\mathcal{S}^{\prime \prime} \subseteq \mathcal{S}$ and an $\mathcal{S}^{\prime \prime \prime} \subseteq \mathcal{S}^{\prime}$ such that $\mathcal{S}^{\prime \prime}$ and $\mathcal{S}^{\prime \prime \prime}$ stand in the basic defeat relation, $\mathcal{S}^{\prime \prime} \sim_{b} \mathcal{S}^{\prime \prime \prime}$.

It's not difficult to verify that this definition lets us recover the defeat relations of the argument framework $\mathcal{F}\left(c_{12}\right)$ from $\mathcal{S}_{4} \sim_{b} \mathcal{S}_{1}$. This is not a coincidence, as our next observation makes clear-its proof is given in the Appendix:

Observation 3.3. Let $c=\langle\mathcal{W}, \mathcal{R}\rangle$ be a context and $\mathcal{S}$ and $\mathcal{S}^{\prime}$ arguments from the argument framework $\mathcal{F}(c)$ constructed from it. Then $\mathcal{S}$ defeats $\mathcal{S}^{\prime}$, according to Definition 11, $\mathcal{S} \leadsto \mathcal{S}^{\prime}$, if and only if $\mathcal{S}$ defeats $\mathcal{S}^{\prime}$, according to Definition 21, $\mathcal{S} \sim_{a} \mathcal{S}^{\prime}$.

Since Definitions 11 and 21 are extensionally equivalent, we can go back and forth between the two ways of thinking about argument frameworks constructed from contexts: we can look at a given framework in its entirety and apply the preference semantics to it to determine its consequences. Alternatively, we can restrict attention to the fragment of this framework that contains only the minimal arguments and apply the semantics to it.

\subsection{Disagreements over disagreement revisited}

Recall the Double Disagreement scenario in which a committed conciliationist is confronted with two disagreeing peers: the metaphysician Milo disagrees with her about the existence of free will, and the epistemologist Evelyn disagrees with her about conciliationism. We expressed this scenario in the context $c_{11}$, depicted again for convenience in Figure 12. Our original model reasoner would derive any formula from it, suggesting that the correct response to the scenario is to conclude everything. But now let's see how the more sophisticated reasoner handles it.

Since $c_{11}$ is a fairly complex context, it'd be difficult to explore the entire argumentation framework $\mathcal{F}\left(c_{11}\right)$ constructed from it. In light of Section 3.3, however, we can restrict attention to a fragment of it that contains only its most informative minimal arguments and the defeat relations among them. This fragment is comprised of: 


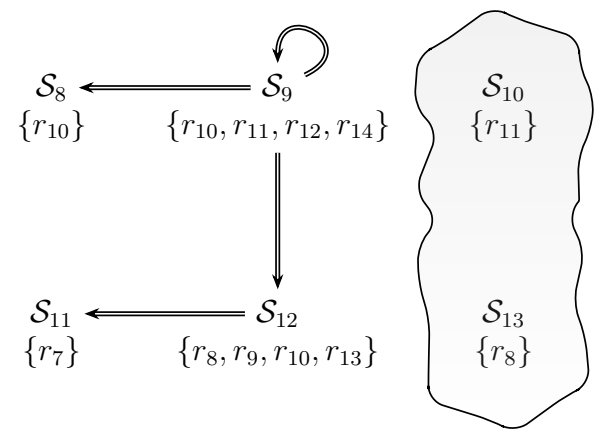

Figure 13: Core arguments from $\mathcal{F}\left(c_{11}\right)$

the $C$-minimal argument $\mathcal{S}_{8}=\left\{r_{10}\right\}$;

the $\operatorname{Out}\left(\mathfrak{r}_{10}\right)$-minimal $\mathcal{S}_{9}=\left\{r_{10}, r_{11}, r_{12}, r_{14}\right\}$, which defeats $\mathcal{S}_{8}$, itself, and $\mathcal{S}_{12}$

the Disagree $(C)$-minimal $\mathcal{S}_{10}=\left\{r_{11}\right\}$;

the $L$-minimal $\mathcal{S}_{11}=\left\{r_{7}\right\}$;

the $\operatorname{Out}\left(\mathfrak{r}_{7}\right)$-minimal $\mathcal{S}_{12}=\left\{r_{8}, r_{9}, r_{10}, r_{13}\right\}$, which defeats $\mathcal{S}_{11}$; and

the Disagree $(L)$-minimal argument $\mathcal{S}_{13}=\left\{r_{8}\right\}$.

The fragment is depicted in Figure 13. The lightly shaded region shows which of its arguments are in the preferred extension of $\mathcal{F}\left(c_{11}\right)$.

It's not difficult to see why the defeat relations obtain: the argument $\mathcal{S}_{9}$ supports the formula $\operatorname{Out}\left(\mathfrak{r}_{10}\right)$, suggesting that the rule $r_{10}$ be taken out of consideration, and, since the arguments $\mathcal{S}_{8}, \mathcal{S}_{9}$ itself, and $\mathcal{S}_{12}$ have this rule as an element, they all come out defeated by $\mathcal{S}_{9}$. Similarly, the argument $\mathcal{S}_{12}$ supports the formula $\mathrm{Out}\left(\mathfrak{r}_{7}\right)$, suggesting that $r_{7}$ be taken out of consideration, and, given that $\mathcal{S}_{11}$ has this rule as an element, it gets defeated by $\mathcal{S}_{12}$. As the picture makes clear, the arguments $\mathcal{S}_{8}$ and $\mathcal{S}_{11}$ are not included in the preferred extension of $\mathcal{F}\left(c_{11}\right)$. Hence, neither $C$, nor $L$ follow from the context, or $c_{11} \psi_{p} C$ and $c_{11} \mu_{p} L$. And this means that our sophisticated reasoner-henceforth the reasoner, without qualification - suggests that the correct response to Double Disagreement is to abandon both the belief in conciliationism and the belief in the existence of free will.

What should we make of this recommendation? The first thing to note is that it's perfectly consistent. Our model captures an extreme version of conciliationism, and yet it does not issue inconsistent recommendations in a paradigm case involving a disagreement over the epistemic significance of disagreement. ${ }^{37}$

\footnotetext{
${ }^{37}$ In fact, our model implements a version of conciliationism that comes close to the infamous Equal Weights View-see, e.g., (Elga 2007).
} 
So, assuming our model captures conciliationism adequately - which, I think, it does - we can conclude that Elga (2010) is mistaken: it's not the case that (all) conciliatory views lead to inconsistency when they turn on themselves.

But the fact that a view doesn't issue inconsistent recommendations does not make it plausible, let alone show that it is correct. And there's reason to feel uneasy about the reasoner's response: it appears to be incoherent. If one is to abandon the belief in conciliationism, why on Earth would one respond to the disagreement over the existence of free will as a conciliationist would? I contend, however, that this is the correct response, and most of what I do in the remainder of this paper will be directed toward explaining why it looks incoherent, but in fact isn't. Before I turn to this task, however, it'll be informative to take a brief look at the constructive responses to the self-defeat objection that have been offered in the literature.

These fall in three categories, depending on how they respond to two crucial questions about the behavior of conciliatory views in cases like Double Disagreement. The first one is whether or not a committed conciliationist ought to abandon her belief in conciliationism in such cases. The second is whether or not she also ought to abandon the belief in free will-or the belief in whatever the first-order disagreement happens to be about - once she has abandoned the belief in conciliatonism. The first category of responses includes those of Bogardus (2009), Christensen (2013), Elga (2010), and Pittard (2015) all of whom reply to the first question in the negative. ${ }^{38}$ The second category is comprised of Matheson's $(2015 \mathrm{a}, \mathrm{b})$ response who replies to the first question in the affirmative and the second one in the negative. These two types of replies - no to the first question and yes to the first and no to the second - were taken to be the only sensible replies up until very recently. But now there is a third category too that includes the responses of Christensen (2021) and Littlejohn (2020) both of whom suggest that there may be nothing wrong with replying to both questions in the affirmative. Littlejohn seems to appeal to burden of proof: as long the opponents of conciliatory views haven't explained what's wrong with the apparently incoherent responses - which they haven't - their advocates don't have anything to worry about. ${ }^{39}$ And Christensen offers a compelling positive argument for the conclusion that the incoherent (or "akratic") response can be rational. ${ }^{40}$

\footnotetext{
${ }^{38}$ The second question doesn't even arise for the responses falling under the first category. It should be clear that anyone who thinks that the belief in conciliatonism doesn't have to be abandoned will be happy saying that the belief in free will has to be.

${ }^{39}$ Littlejohn's stance appears very clearly in the following passage: "..a conciliatory thinker can continue to suspend when peers disagree without having any attitudes at all towards [conciliationism]. The two things recommended (i.e., being conciliatory on some contested propositions, suspending on [conciliationism]) are perfectly possible to do together. Thus, they aren't incompatible. The simple [self-defeat] objections simply misses its intended target" (Littlejohn 2020, p. 1382). I interpret Littlejohn as appealing to burden of proof because his defense of the described responses is confined to drawing an analogy with the practical domain. For completeness, I should add that his main focus is not the "simple" self-defeat objection we have been discussing, but the "subtle" one. We don't need to worry about the latter one here.

${ }^{40}$ Contrary to the earlier position defended in (Christensen 2013), Christensen no longer
} 
Thus, our formal analysis points to the same response as the most recent replies to the self-defeat objection. This suggests that we may well be on the right track, even if the reasoner's response may seem odd to us. Now, I think that the reason it seems odd to us stems from the fact that the story recounted in Double Disagreement is underdescribed. Notice that it's very natural to think that the answer to the question of which response to the scenario is rational depends, in part, on the agent's rational degrees of confidence in the conclusions of her (first-order) reasoning about conciliatiationism, free will, and the disagreements over these two matters. ${ }^{41}$ If the agent is much more confident in the reasoning that led her to conclude that she and Milo are in genuine disagreement than in the reasoning that led her to conclude that free will exists, then, intuitively, she should abandon her belief in free will. If, by contrast, she is much more confident in the reasoning that led her to conclude that free will exists than in the reasoning that led her to conclude that there's genuine disagreement over it, then, intuitively, it's fine for her to retain the belief in free will. ${ }^{42}$ So the agent's degrees of confidence can make a difference for how she is to respond to Double Disagreement, and yet its description doesn't provide us with enough detail to figure out what the agent's relevant degrees of confidence are. ${ }^{43}$

This observation supports two conclusions. First, given the importance of degrees of confidence, any fully adequate model of conciliatory reasoning should be able to accommodate them, and so our model, in particular, needs to be modified further. And second, given that the description doesn't fully specify all normatively-relevant details, we shouldn't expect there to be only one context representing Double Disagreement and only one rational response to it. Instead, we should expect that there are going to be many such contexts - representing various versions of the scenario in which different degrees of confidence (in the reasoning about conciliationism, free will, and the disagreements about them) are rational - and that they won't necessarily call for the same rational response.

thinks that the correct response to Double Disagreement leads to a violation of some epistemic ideal. He seems to think that there aren't any ideals that an agent in an akratic state would violate, that there are plenty of cases where it's rational for one to believe $X$ while doubting the rationality of that very belief, and that, without assuming that this is never rational, the self-defeat objection doesn't really get off the ground.

${ }^{41}$ It's worth emphasizing that, when I talk about degrees of confidence, I mean rational degrees of confidence, or degrees of confidence that are justified in the agent's epistemic situation, or rational for the agent to have given her epistemic situation-compare to Lackey's (2010a, 2010b) "degrees of justified confidence", and Lasonen-Aarnio's (2013) "correct credences". These shouldn't be confused with phenomenal feelings of confidence.

${ }^{42} \mathrm{Cf}$. Matheson (2015a) who suggests that whether or not conciliationism turns on itself in a case like Double Disagreement and, thus, whether or not the belief in it should be abandoned depends on the details of the case.

${ }^{43}$ One might worry that I'm making the scenario more ambiguous than it is and say that it's pretty clear from its description that the agent is at least as confident in the reasoning suggesting that her disagreement with Milo is genuine as the reasoning suggesting that free will exists. In response, even if that's so, not much hinges on it: I used these particular degrees of confidence here because they make for the simplest illustration. As will become clear in due time, the agent's relative degrees of confidence in her reasoning about conciliationism and free will can also make a difference for how she should respond to the scenario, and the description certainly doesn't provide enough detail for us to say what these degrees are. 
In light of this, it shouldn't be surprising that by embedding Double Disagreement in the context $c_{11}$ we have implicitly filled in the missing information about the agent's degrees of confidence in a particular way. Once we make this information explicit, the reasoner's response will look much more plausible, or so I'm going to argue.

\section{Adding degrees of confidence}

This section has two main goals. The first is to extend our model reasoner, so that it is sensitive to the information about (rational) degrees of confidence. The second is to explore how the agent's degrees of confidence might determine what's rational for her to do in Double Disagreement. As flagged, this will shed light on the seemingly incoherent response we saw above.

It's natural to expect that degree-of-confidence talk will go together with numerical values. In the present context, however, degrees of confidence will be represented not by means of numerical values, but, rather, by means of a comparative relation. This means that it won't make sense to ask about one's degree of confidence in some proposition unless it's compared to one's degree of confidence in another proposition, or that we'll always be concerned only with relative degrees of confidence.

The rest of this section is structured as follows. Section 4.1 is concerned with relative degrees of support (not confidence): it introduces the idea of a priority relation over rules of a context and shows how it can be used to relativize the relation of support between arguments and their conclusions. Once this is done, it won't necessarily hold that any two arguments support their conclusions to the same degree. Instead, what will typically happen is that one argument supports its conclusion to a greater degree, or more strongly, than another argument supports its conclusion. Section 4.2 returns to Double Disagreement, explains how the relevant degrees of confidence - that is, the agent's degrees of confidence in the reasoning about conciliationism, free will, and the disagreements about these two questions - can be mapped on the priority relation over rules, and works through an illustrative example. Finally, Section 4.3 provides a general answer to the question of how do the reasoner's responses depend on what the rational degrees of confidence in Double Disagreement are, situates this answer in the literature, and revisits the seemingly incoherent response from Section 3.4 .

\subsection{Relativizing support to degrees of support}

Our model reasoner determines the formulas that follow from a given context roughly as follows. It starts by constructing an argument framework from the context, then it selects its set of winning arguments, and, finally, it outputs the conclusions this set supports. However, throughout this process, the reasoner takes all arguments to support their conclusions equally well, or to the same degree. The first step toward factoring degrees of confidence in the model is to 
relativize support to degrees of support. This will have a direct effect on how conflicts between arguments get resolved and, thus, on which set of arguments comes out winning.

The idea that (defeasible) arguments can support their conclusions to varying degrees, and that this can affect resolution of conflicts between arguments is both natural and familiar. ${ }^{44}$ We're going to implement this idea in our model, drawing on two principles I call Weakest Link and Winner Takes All, both of which come from Pollock's $(1995,2001,2010)$ work.

Before we turn to these principles, we need to extend contexts with a priority relation on rules. Let the statement $r \leq r^{\prime}$ mean that the premise of the rule $r^{\prime}$, Premise $\left[r^{\prime}\right]$, confers at least as much support on its conclusion, Conclusion $\left[r^{\prime}\right]$, as the premise of the rule $r$, Premise $[r]$, confers on its respective conclusion, Conclusion $[r]$. For the sake of brevity, in what follows we often omit the reference to rule premises and conclusions, reading $r \leq r^{\prime}$ as saying, simply, that $r^{\prime}$ is at least as strong as $r$. We also require that $\leq$ satisfies some natural properties. First, it must be reflexive, meaning that each rule must be at least as strong as itself:

$$
r \leq r .
$$

Second, it must be transitive, meaning that whenever $r^{\prime}$ is at least as strong as $r$ and $r^{\prime \prime}$ is at least as strong as $r^{\prime}$, the rule $r^{\prime \prime}$ has to be at least as strong as $r$ :

$$
\text { if } r \leq r^{\prime} \text { and } r^{\prime} \leq r^{\prime \prime} \text {, then } r \leq r^{\prime \prime} \text {. }
$$

Third, the relation $\leq$ must satisfy connectivity, which says that any two rules can be compared with respect to their strength:

$$
r \leq r^{\prime}, r^{\prime} \leq r \text { or both. }
$$

Requiring that all rules are comparable should make good sense here: for any two considerations conferring support to different conclusions, we'd seem to always be able to ask which of the two confers more. ${ }^{45}$ It'll also be useful to introduce some shorthand notation: when we have $r \leq r^{\prime}$ without $r^{\prime} \leq r$, we write $r<r^{\prime}$. And when we have both $r \leq r^{\prime}$ and $r^{\prime} \leq r$ (for distinct rules), we write $r \sim r^{\prime}$.

In the remainder of this paper, we will be concerned with weighted contexts:

Definition 22 (Weighted contexts). A weighted context $c$ is a structure of the form $\langle\mathcal{W}, \mathcal{R}, \leq\rangle$ where $\langle\mathcal{W}, \mathcal{R}\rangle$ is a context and $\leq$ is a reflexive, transitive, and connected relation (or a connected preorder) on $\mathcal{R}$.

\footnotetext{
${ }^{44}$ See, e.g., (Dunne et al. 2011), (Grossi \& Modgil 2015), (Modgil \& Prakken 2013), (Pollock 2001, 2010), and (Prakken \& Sartor 1997).

${ }^{45}$ Compare to (Horty 2012, Section 1.1.2) who opts for a strict partial order-as opposed to a weak preorder, as we do - and explicitly rejects connectivity. It should be noted, though, that Horty works in a different context, using default logic to model reasons and reason interaction in different domains. Pollock $(1994,1995,2001)$ sticks to the epistemic domain and requires connectivity, just as we do here.
} 


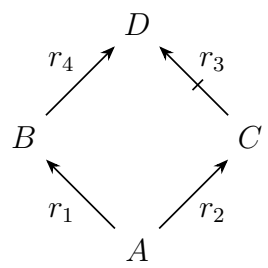

$r_{1}<r_{2}<r_{3}<r_{4}$

Figure 14: Weakest Link Principle

As our first example, consider the context $c_{14}=\langle\mathcal{W}, \mathcal{R}, \leq\rangle$ with $\mathcal{W}=\{A\}, \mathcal{R}$ $=\left\{r_{1}=\frac{A}{B}, r_{2}=\frac{A}{C}, r_{3}=\frac{C}{\neg D}, r_{4}=\frac{B}{D}\right\}$, and $r_{1}<r_{2}<r_{3}<r_{4} \cdot{ }^{46}$ The ordering tells us that the rule $r_{4}$ is the strongest, that it's followed by $r_{3}$, then by $r_{2}$, and that $r_{1}$ is the weakest. Figure 14 represents this context graphically.

Now let's zoom in on two arguments based on $c_{14}$, namely, $\mathcal{S}_{1}=\left\{r_{1}, r_{4}\right\}$ and $\mathcal{S}_{2}=\left\{r_{2}, r_{3}\right\}$. Since we have $\mathcal{W} \cup$ Conclusion $\left[\mathcal{S}_{1}\right] \vdash D$ and $\mathcal{W} \cup$ Conclusion $\left[\mathcal{S}_{2}\right] \vdash$ $\neg D$, these two arguments support opposing conclusions. Given that the four rules now have relative weights, we can ask about the support that each of these arguments confers on their conclusion. According to our next definition, their relative degrees of support are determined using the Weakest Link Principle. ${ }^{47}$

Definition 23 (Argument Strengths, using Weakest Link). Let $c=\langle\mathcal{W}, \mathcal{R}, \leq\rangle$ be a weighted context and $\mathcal{S}$ and $\mathcal{S}^{\prime}$ two scenarios based on it. Then $\mathcal{S}^{\prime}$ is at least as strong as $\mathcal{S}$, written as $\mathcal{S} \leq \mathcal{S}^{\prime}$, if and only if there is a rule $r$ in $\mathcal{S}$ such that, for all $r^{\prime} \in \mathcal{S}^{\prime}, r \leq r^{\prime}$.

As its name suggests, the Weakest Link Principle says that an argument is exactly as strong as its weakest element. With regard to $\mathcal{S}_{1}$ and $\mathcal{S}_{2}$, in particular, it says that $\mathcal{S}_{1}$ is only as strong as $r_{1}$, that $\mathcal{S}_{2}$ is only as strong as $r_{2}$, and that, therefore, $\mathcal{S}_{2}$ supports $\neg D$ to a greater degree than $\mathcal{S}_{1}$ supports $D$. Notice how this happens: since $\mathcal{S}_{1}$ contains the rule $r_{1}$ that's (strictly) weaker than both rules in $\mathcal{S}_{2}$ - that is, we have both $r_{1}<r_{2}$ and $r_{1}<r_{3}$ - the argument $\mathcal{S}_{2}$ is at least as strong as $\mathcal{S}_{1}$, or $\mathcal{S}_{1} \leq \mathcal{S}_{2}$. But even though $\mathcal{S} \leq \mathcal{S}^{\prime}$ doesn't exclude the possibility that $\mathcal{S}^{\prime} \leq \mathcal{S}$ holds, in the particular case at hand this doesn't happen. While $r_{4}$ is stronger than both elements of $\mathcal{S}_{2}$, its other element $r_{1}$ is (strictly) weaker than both of them. Thus, there's no rule in $\mathcal{S}_{2}$ that would be only as

\footnotetext{
${ }^{46}$ We can assume that the reasoner deems every rule in $\mathcal{R}$ prima facie reasonable to follow from the outset and ignore the Reasonable-formulas and the reasonableness constraint in this section. Nothing important hinges on this.

${ }^{47}$ Notice that the definition lifts the relation between rules to sets containing those rules. There are many alternative ways of lifting a relation between elements to sets containing those elements - see (Barberà et al. 2004) for a thorough survey. The question of how our analysis might change if we used a different lifting operation-perhaps, that of Brass (1991) or Horty (2012) — must be left for future work.
} 


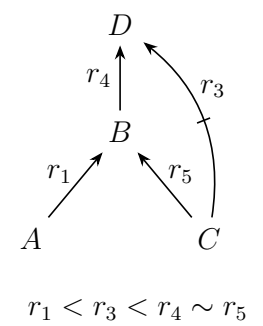

Figure 15: Minimal arguments and Weakest Link

strong as all the rules in $\mathcal{S}_{1}$. So we have $\mathcal{S}_{1} \leq \mathcal{S}_{2}$ and not $\mathcal{S}_{2} \leq \mathcal{S}_{1}$, or $\mathcal{S}_{1}<\mathcal{S}_{2}$ in the shorthand.

Notice that Definition 23 lets us compare any two scenarios. Our next example illustrates why this may seem problematic. In Section 3.3, we looked at the context $c_{13}$ with $\mathcal{W}=\{A, C\}$ and $\mathcal{R}$ consisting of the rules $r_{1}=\frac{A}{B}$, $r_{4}=\frac{B}{D}$, and $r_{5}=\frac{C}{B}$. Now we extend it in two ways. First, we supplement $\mathcal{R}$ with the rule $r_{3}=\frac{C}{\neg D}$. Second, we add an ordering on $\mathcal{R} \cup\left\{r_{3}\right\}$, namely, $r_{1}<r_{3}<r_{4} \sim r_{5}$. The result is the weighted context $c_{15}=\left\langle\mathcal{W}, \mathcal{R} \cup\left\{r_{3}\right\}, \leq\right\rangle$, depicted in Figure 15.

Let's zoom in on the arguments $\mathcal{S}_{3}=\left\{r_{1}, r_{4}, r_{5}\right\}$ and $\mathcal{S}_{4}=\left\{r_{3}\right\}$ based on $c_{15}$. The first supports the formula $D$, the second supports the contrary formula $\neg D$. And it's easy to see that, on Definition $23, \mathcal{S}_{4}$ comes out stronger than $\mathcal{S}_{3}$ : there's a rule in $\mathcal{S}_{3}$, namely, $r_{1}$, that is weaker than every rule in $\mathcal{S}_{4}$. This verdict may seem counternintuitive, since $\mathcal{S}_{3}$ allows one to reach $D$ without relying on the relatively weak rule $r_{1}$ at all, using the chain $r_{5}-r_{4}$ whose elements are both (strictly) stronger than $r_{3}$. However, the fault here doesn't lie with Definition 23, but, rather, with the fact that we are juxtaposing $\mathcal{S}_{3}$ and $\mathcal{S}_{4}$. The argument $\mathcal{S}_{3}$ actually combines two $D$-minimal arguments, namely, $\mathcal{S}_{5}=\left\{r_{1}, r_{4}\right\}$ and $\mathcal{S}_{6}=\left\{r_{4}, r_{5}\right\}$. When we juxtapose $\mathcal{S}_{5}$ and $\mathcal{S}_{6}$ with $\mathcal{S}_{4}$, we get the intuitive result - or the result we'd expect the Weakest Link to deliver - namely, that $\mathcal{S}_{5}<\mathcal{S}_{4}$ and $\mathcal{S}_{4}<\mathcal{S}_{6}$. Since what's important for whether $D$ does or doesn't follow from the context $c_{15}$ is whether or not there's some argument supporting $D$ that's not undermined by the rule $r_{3}$, the definition does just fine.

Although the Weakest Link tells us how to compare strengths of arguments, it doesn't tell us how to resolve conflicts between arguments. This is why we need a second principle. Intuitively, the conflict between $\mathcal{S}_{1}$ and $\mathcal{S}_{2}$ should be resolved in favor of the stronger argument $\mathcal{S}_{2}$. However, an important question remains: what's the all-things-considered degree of support conferred on $\neg D$, or how well is it supported after all the relevant information has been taken into consideration? There seem to be two prima facie reasonable answers to this question. According to the first, all-things-considered degree of support conferred on $\neg D$ equals the degree of support that $\mathcal{S}_{2}$ confers on $\neg D$. According 


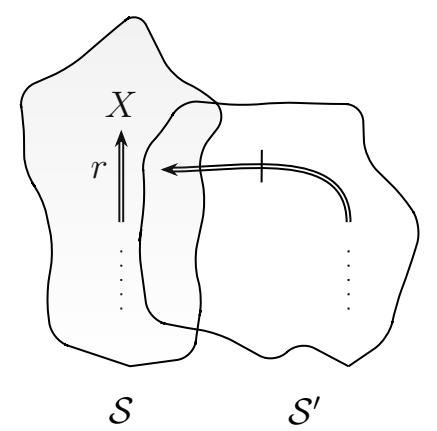

Figure 16: Winner Takes All and exclusionary rules

to the second, the degree of support that $\mathcal{S}_{2}$ confers on $\neg D$ is to be taken as a starting point and it is to be attenuated, in one way or another, factoring in the strength of the contrary argument $\mathcal{S}_{1}$. I'm going to adopt the first response here, or the response that goes with the Winner Takes All Principle. This is not because I have a knockdown argument against the alternative, but because I have no idea how to capture the alternative formally. ${ }^{48}$

Also, note that Winner Takes All doesn't only let us resolve conflicts between arguments supporting contrary conclusions, but can also determine if the support that an argument confers on its conclusion gets undermined by some other argument: suppose that we have two arguments $\mathcal{S}$ and $\mathcal{S}^{\prime}$, that $\mathcal{S}$ supports some formula $X$, and that $\mathcal{S}^{\prime}$ contests the support that $\mathcal{S}$ confers on $X$ by supporting the proposition $O u t(\mathfrak{r})$, with $r$ being a rules that the support $\mathcal{S}$ lends to $X$ crucially depends on - see Figure 16 for a schematic representation. Applying the principle here amounts to saying that $\mathcal{S}^{\prime}$ cancels all the support that $\mathcal{S}$ confers on $X$ if $\mathcal{S}^{\prime}$ is at least as strong as $\mathcal{S}$, and that it has no effects otherwise. $^{49}$

Our next definition, then, specifies a notion of defeat, taking into account varying degrees of support and drawing on the two principles. The first is man-

\footnotetext{
${ }^{48}$ The recent work on the so-called numerical argumentation frameworks - see, e.g., (Barringer et al. 2012) and (Gabbay 2012) - might prove useful in formalizing the second response. However, this work is still in its infancy stage, and many complex issues have to get resolved before it would be possible to apply it in the present context. Chief among them is the question of how to assign numerical weights when the underlying network, or inference graph, contains cycles - see (Barringer et al. 2012, Secs. 3-4). Also, Pollock does offers an interesting argument against the idea that the strengths of winning arguments should be attenuated by the losing ones: he thinks that it commits one to (unrestricted) accrual of reasons, or the view that two arguments to the same conclusion can confer a higher degree of support on it than either of the arguments alone, and that there are good independent reasons to reject this view-see, e.g., (Pollock 1995, pp. 101-4).

${ }^{49}$ It's worth mentioning that there's an alternative to Winner Takes All. We could let $\mathcal{S}^{\prime}$ do its destructive work no matter how it compares to $\mathcal{S}$. Pollock consider this possibility and rejects it on the basis of its being "perverse" - see (Pollock 1995, pp. 103-4). But see (Horty 2012, pp. 204-10) for a response.
} 
ifest in the definition's reliance on the $\leq$ relation. The second in its binary character, or the fact that a potential defeater $\mathcal{S}$ of $\mathcal{S}^{\prime}$ either fully defeats $\mathcal{S}^{\prime}$, or has absolutely no effect on it.

Definition 24 (Defeat with degrees). Let $c=\langle\mathcal{W}, \mathcal{R}, \leq\rangle$ be a weighted context and $\mathcal{S}$ and $\mathcal{S}^{\prime}$ two arguments based on it. Then $\mathcal{S}$ defeats $\mathcal{S}^{\prime}$, written as $\mathcal{S} \leadsto \leq \mathcal{S}^{\prime}$, if and only if there is some rule $r \in \mathcal{S}^{\prime}$ such that either $\mathcal{W} \cup$ Conclusion $[\mathcal{S}] \vdash$ $\neg$ Conclusion $[r]$, or $\mathcal{W} \cup$ Conclusion $[\mathcal{S}] \vdash \operatorname{Out}(\mathfrak{r})$ and $\mathcal{S}^{\prime} \leq \mathcal{S}$.

There's only one difference between this definition and Definition 11 (Defeat) from Section 3.1, namely, the requirement that the defeating argument is at least as strong as the the defeated one. An easy check will convince you that this definition delivers the intuitive results in the examples that we looked at.

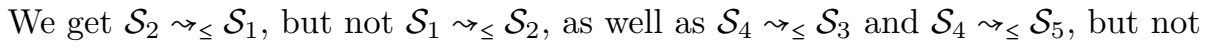
$\mathcal{S}_{4} \leadsto \leq \mathcal{S}_{6}$.

With the new definition of defeat in hand, we can construct argument frameworks from weighted contexts:

Definition 25 (Argument frameworks based on weighted contexts). Let $c=$ $\langle\mathcal{W}, \mathcal{R}, \leq\rangle$ be some weighted context. Then the argument framework $\mathcal{F}(c)$ based on $c$ is the pair $\left\langle\mathcal{A}, \sim_{\leq}\right\rangle$where $\mathcal{A}$ is the set Arguments(c) and $\sim_{\leq}$is the set $\left\{\left(\mathcal{S}, \mathcal{S}^{\prime}\right) \in \mathcal{A} \times \mathcal{A}: \mathcal{S} \leadsto_{\leq} \mathcal{S}^{\prime}\right\}$.

Stability and preference semantics can be applied to argument frameworks, whether they be built from regular or weighted contexts. This means that there's no need to change the definitions from Section 3.2. Also, it's straightforward to define the analogue of basic defeat from Section 3.3, taking into account the relative strengths of rules, as well as an alternative procedure for acquiring argument frameworks based on it. But we won't do it here for reasons of space. We close this section with an observation, showing that the addition of weights to contexts is a conservative generalization of our original model - its proof can be found in the Appendix:

Observation 4.1. Let $c=\langle\mathcal{W}, \mathcal{R}\rangle$ be a regular context and $c^{\prime}=\langle\mathcal{W}, \mathcal{R}, \leq\rangle$ be the same context with a connected preorder $\leq$ assigning all the rules $r$ in $\mathcal{R}$ the same weight-so, for all $r, r^{\prime} \in \mathcal{R}, r \sim r^{\prime}$. Then $\mathcal{F}(c)=\mathcal{F}\left(c^{\prime}\right)$.

\subsection{From degrees of confidence to degrees of support}

Having developed some new tools, we can return to Double Disagreement. We expressed it in the context $c_{11}=\langle\mathcal{W}, \mathcal{R}\rangle$, which is depicted in Figure 17 one final time. In Section 3.4, we noted that it needs to be supplemented with the information about the agent's degrees of confidence in the conclusions of her domain-specific reasoning. Now recall which propositions the formulas $\operatorname{Seems}(C)$, Seems $(L)$, Seems(Disagree $(C))$, and Seems(Disagree $(L))$ express. They are, respectively, that the agent's (first-order) reasoning suggests that conciliationism is true, that her reasoning suggests that we have free will, 


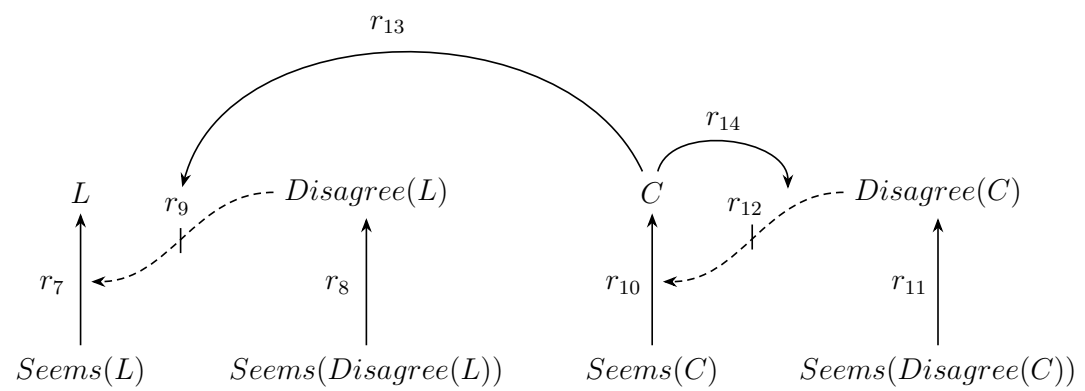

Figure 17: Double Disagreement, once more

that her reasoning suggests that she's in genuine disagreement over conciliationism, and that it suggests she's in genuine disagreement over free will. In light of this, it's very natural to associate the agent's degrees of confidence with these formulas.

However, given that, in weighted contexts, weights are associated with rules, and not formulas, we need to express the degrees of confidence associated with the Seems-formulas in terms of a priority relation on rules. As a first step, notice that every rule in $c_{11}$ is of one of three forms, namely, $r(X)=\frac{\operatorname{Seems}(X)}{X}$, $r^{\prime}(X)=\frac{\text { Disagree }(X)}{\text { Out }(\mathfrak{r}(X))}$, or $\frac{C}{\text { Reasonable }\left(\mathfrak{r}^{\prime}(X)\right)}$. Now notice that the (relative) strengths of the rules fitting the first form correspond directly to the (relative) degrees of confidence associated with the Seems-formulas. Thus, suppose we want to capture a version of Double Disagreement where the agent is least confident in the reasoning leading her to conclude that she's in genuine disagreement over conciliationism, Seems $(\operatorname{Disagree}(C))$, more confident in the reasoning leading her to conclude that she's in genuine disagreement over free will, Seems(Disagree $(L)$ ), even more confident in her reasoning about free will, Seems $(L)$, and most confident in her reasoning about conciliationism, Seems $(C)$. Here we'd order the four rules instantiating the $r(X)$-schema as follows: $r_{11}<r_{8}<r_{7}<r_{10}$.

Turning to the rules that don't fit this schema, notice that every argument based on $c_{11}$ containing either the rule $r_{13}=\frac{C}{\operatorname{Reasonable}\left(\mathfrak{r}_{9}\right)}$ or the rule $r_{14}=\frac{C}{\text { Reasonable }\left(\mathfrak{r}_{12}\right)}$ has to contain $r_{10}=\frac{\operatorname{Seems}(C)}{C}$, and that every argument containing $r_{9}=\frac{\operatorname{Disagree}(L)}{\operatorname{Out}\left(\mathfrak{r}_{7}\right)}$ or $r_{12}=\frac{\operatorname{Disagree}(C)}{\operatorname{Out}\left(\mathfrak{r}_{10}\right)}$ has to contain $r_{10}$ too, as well as at least one additional rule of the form $\frac{\operatorname{Seems}(\operatorname{Disagree}(X))}{\operatorname{Disagree}(X)}$. This is due to the structure of $c_{11}$ and the fact that all rules comprising an argument have to be triggered and deemed reasonable in it. For illustration, consider 
some scenario $\mathcal{S}$ based on $c_{11}$ that contains the rule $r_{13}=\frac{C}{\operatorname{Reasonable}\left(\mathfrak{r}_{9}\right)}$. Does $\mathcal{S}$ qualify as an argument based on $c_{11}$ ? Well, it can qualify only in case $r_{13}$ is triggered in it, and, for $r_{13}$ to be triggered in it, $\mathcal{S}$ has to contain $r_{10}$. This is important because our model reasoner relies on the Weakest Link Principle to determine the relative strengths of arguments. One direct consequence is that any argument based on $c_{11}$ can be only as strong as the rules of the form $\frac{\operatorname{Seems}(X)}{X}$ it contains. Thus, there's a clear sense in which what really matters for the relative strengths of arguments - and, thus, also for the overall conclusions the reasoner draws - are the relative strengths of the four rules that have this form, namely, $r_{7}, r_{8}, r_{10}$, and $r_{11}$.

What's more, on a certain plausible assumption, the relative strengths of these rules are all that matters. We can see what's at stake here by looking at the argument $\mathcal{S}_{7}=\left\{r_{10}=\frac{\operatorname{Seems}(C)}{C}, \quad r_{13}=\frac{C}{\operatorname{Reasonable}\left(\mathfrak{r}_{9}\right)}\right\}$. Because of the Weakest Link Principle, the support that $\mathcal{S}_{7}$ confers on Reasonable $\left(\mathfrak{r}_{9}\right)$ can be only as strong as $r_{10}$. It is, however, possible for this support to be much lower, and it will be lower in all cases where the strength of $r_{13}$ is lower than that of $r_{10}$. That's why we are going to rule out such cases, assuming that the following principle holds true:

No Support Lost: The relative weights of rules whose form is either

$$
\begin{aligned}
& \frac{\text { Disagree }(X)}{\operatorname{Out}(\mathfrak{r}(X))} \text { or } \frac{C}{\text { Reasonable }\left(\mathfrak{r}^{\prime}(X)\right)} \text { must be at least as high as the } \\
& \text { weights of rules of form } \frac{\operatorname{Seems}(X)}{X} \text { that they depend on. }
\end{aligned}
$$

The intuitive idea of dependency between rules which this assumption appeals to can be made precise in terms of the relations between the arguments based on $c_{11}$. If there's no argument $\mathcal{S}$ based on $c_{11}$ that contains $r$ but not $r^{\prime}$, then $r$ depends on $r^{\prime}$. Notice that No Support Lost formalizes the intuitive idea that the strengths of the arguments based on $c_{11}$ should depend only on the agent's relevant degrees of confidence. ${ }^{50}$ So it looks like a perfectly reasonable assumption.

With No Support Lost in place, there's a general answer to the question of how do the reasoner's responses depend on the relative strengths of the four crucial rules. The next section stars with this answer. The remainder of this one works through an example, illustrating how the addition of an ordering on rules in $c_{11}$ affects the reasoner's response.

Let $c_{16}=\langle\mathcal{W}, \mathcal{R}, \leq\rangle$ be the weighted context we acquire by extending $c_{11}$ with the ordering $r_{11} \sim r_{12}<r_{9} \sim r_{10} \sim r_{13} \sim r_{14}<r_{7}<r_{8}$. It expresses a version of Double Disagreement where the agent is least confident in the reasoning leading her to conclude that the disagreement over conciliationism is genuine,

\footnotetext{
${ }^{50}$ We'll soon see how the reasoner's recommendations depend on the relevant degrees of confidence. Without No Support Lost, the reasoner's recommendations still depend on degrees of confidence, but the correlations are more messy.
} 


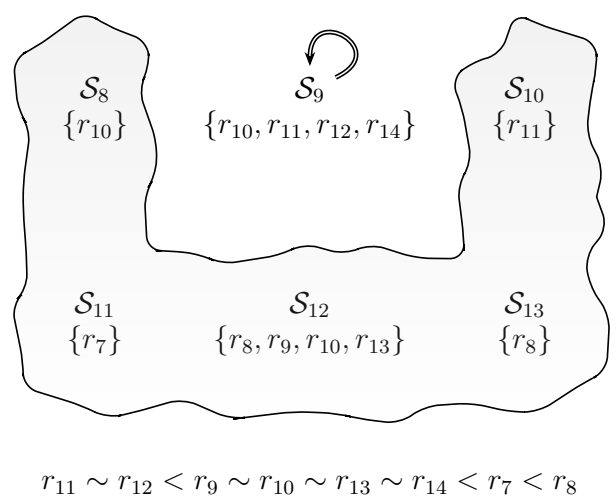

Figure 18: Core arguments from $\mathcal{F}\left(c_{16}\right)$

Seems(Disagree $(C))$, more confident in the reasoning leading her to conclude that conciliationism is true, Seems $(C)$, even more confident in the reasoning suggesting that free will exists, Seems $(L)$, and most confident in the reasoning suggesting that the disagreement over free will is genuine, Seems(Disagree $(L))$. Notice that the ordering assigns the rules whose form is either $\frac{\operatorname{Disagree}(X)}{\operatorname{Out}(\mathfrak{r}(X))}$ or $\frac{C}{\text { Reasonable }\left(\mathfrak{r}^{\prime}(X)\right)}$ the same relative weights as the weakest rules of the form $\frac{\operatorname{Seems}(X)}{X}$ that they depend on. This is only for the sake of simplicity. Nothing would change if these rules were assigned different relative weights, as long as No Support Lost wasn't violated.

We restrict attention to a fragment of the argument framework $\mathcal{F}\left(c_{16}\right)$, containing its most informative minimal arguments and the defeat relations between them - this is the same fragment we looked at when considering the framework $\mathcal{F}\left(c_{11}\right)$ in Section 3.4:

the $C$-minimal argument $\mathcal{S}_{8}=\left\{r_{10}\right\}$;

the $\operatorname{Out}\left(\mathfrak{r}_{10}\right)$-minimal $\mathcal{S}_{9}=\left\{r_{10}, r_{11}, r_{12}, r_{14}\right\}$, which defeats itself;

the Disagree $(C)$-minimal $\mathcal{S}_{10}=\left\{r_{11}\right\}$;

the $L$-minimal $\mathcal{S}_{11}=\left\{r_{7}\right\}$;

the $\operatorname{Out}\left(\mathfrak{r}_{7}\right)$-minimal $\mathcal{S}_{12}=\left\{r_{8}, r_{9}, r_{10}, r_{13}\right\}$; and

the Disagree $(L)$-minimal argument $\mathcal{S}_{13}=\left\{r_{8}\right\}$.

The fragment is depicted in Figure 18. Notice that there are fewer defeat relations between arguments, when compared to $\mathcal{F}\left(c_{11}\right)$. There the self-defeating 
argument $\mathcal{S}_{9}$ defeated both $\mathcal{S}_{8}$ and $\mathcal{S}_{12}$. Now it does not, and it's easy to see why: since $\mathcal{S}_{9}$ contains $r_{11}$ and $r_{12}$ that are strictly weaker than the only element of $\mathcal{S}_{8}$, namely, $r_{10}$, it's not the case that $\mathcal{S}_{8} \leq \mathcal{S}_{9}$. And that's a prerequisite for $\mathcal{S}_{9}$ to defeat $\mathcal{S}_{8}$. Similarly, the $\operatorname{Out}\left(\mathfrak{r}_{7}\right)$-minimal $\mathcal{S}_{12}$ no longer defeats the $L$-minimal $\mathcal{S}_{11}$. Yet again, $r_{7}$ is stronger than some of the elements of $\mathcal{S}_{12}$, and so $\mathcal{S}_{11} \leq \mathcal{S}_{12}$ doesn't hold.

Since $\mathcal{S}_{8}$ and $\mathcal{S}_{11}$ are in the preferred extension of $\mathcal{F}\left(c_{16}\right)$ - the shaded region in Figure 18 - both $C$ and $L$ follow from $c_{16}$. So the reasoner suggests that, in the particular version of Double Disagreement that is captured by $c_{16}$, one should stick to one's belief in conciliationism, as well as one's belief in free will. At first blush, this recommendation might look incoherent. Since the agent retains the belief in conciliationism, why wouldn't she conciliate in response to the disagreement over free will - especially, given the fact that she's more confident in it being genuine than her own reasoning about free will?

We shouldn't forget, however, that we're dealing with a situation where the agent is more confident in the reasoning that led her to conclude that we have free will than the reasoning that led her to conclude that conciliationism is correct. And it's not difficult to imagine her reasoning as follows:

After deliberating about the epistemic significance of disagreement, conciliationism seems to me to be correct. If it is, I should abandon my belief in the existence of free will in response to my disagreement with Milo. However, I've more faith in my deliberation about the question of free will than my deliberation about the epistemic significance of disagreement, and it would be foolish of me to abandon a view on the basis of another view that seems to me to be correct, but that I'm also less confident of.

It's not only that there's nothing incoherent about this line of thought. It also seems very intuitive. So the reasoner's response to $c_{16}$ is perfectly reasonable.

\subsection{Disagreements over disagreement with degrees}

Now we can turn to the following question: how does the reasoner's response depend on the degrees of confidence in the conclusions of first-order reasoning? It'll be useful to introduce some simple notation. Recall that $\operatorname{Seems}(X)$ says that the agent has arrived at the conclusion that $X$ after deliberating about whether $X$ to the best of her ability. Now, let $\operatorname{Seems}(X) \leq \operatorname{Seems}(Y)$ express the idea that the agent is at least as confident in the conclusion of her reasoning about whether $Y$ as she is in the conclusion of her reasoning about whether $X$; and let $\operatorname{Seems}(X)<\operatorname{Seems}(Y)$ be shorthand for $\operatorname{Seems}(X) \leq \operatorname{Seems}(Y)$ and not $\operatorname{Seems}(Y) \leq \operatorname{Seems}(X)$.

As far as the belief in conciliationism is concerned, the reasoner's response depends on the degrees of confidence in $\operatorname{Seems}(C)$ and Seems(Disagree $(C)$ ): it abandons this belief, if the degree of confidence in the truth of conciliationism is only as high as the degree of confidence in the disagreement over 
it being genuine - that is, if $\operatorname{Seems}(C) \leq \operatorname{Seems}(\operatorname{Disagree}(C)$ ) - and retains it otherwise - that is, when $\operatorname{Seems}(C)>\operatorname{Seems}(\operatorname{Disagree}(C))$. This response seems perfectly intuitive. It's also well in line with the literature, in the following sense: those authors who think that conciliatory views can turn on themselves, but also that this isn't fatal for them-that is, Christensen (2021), Littlejohn (2020), and Matheson (2015a,b) - would agree that, in such situations, the belief in conciliationism should be abandoned. ${ }^{51}$ As for the belief in free will, the reasoner's response here depends on the relative degrees of confidence in Seems $(C)$, Seems $(L)$, and Seems(Disagree $(L))$ : it abandons this belief, in case the degree of confidence in the reasoning leading to the conclusion that free will exists is only as high as both the degree of confidence in the disagreement over it being genuine and the degree of confidence in the truth of conciliationism - that is, in case both $\operatorname{Seems}(L) \leq \operatorname{Seems}($ Disagree $(L))$ and $\operatorname{Seems}(L) \leq \operatorname{Seems}(C)$ - and retains the belief otherwise - that is, if either $\operatorname{Seems}(L)>\operatorname{Seems}(\operatorname{Disagree}(L))$, or $\operatorname{Seems}(L)>\operatorname{Seems}(C)$.

Although the reasoner's response with regard to the belief in free will is perfectly reasonable - or so I shall argue - it may go against pre-theoretic intuitions about the behavior of conciliatory views and what's made of their behavior in much of the literature. Three features, in particular, make the reasoner's response surprising - and the first two are independent of the self-defeat objection. The first is that the question of whether it's rational to retain the belief in free will turns out to depend on the relative degrees of confidence in the reasoning that led the agent to conclude that free will exists and the reasoning that led her to conclude that the disagreement over free will is genuine, $\operatorname{Seems}(L)$ and Seems(Disagree $(L))$. In particular, it turns out that, in some cases, a committed conciliationist can rationally retain her belief in free will even if she has a high degree of confidence that there's a genuine disagreement over it. At first blush, this might look like a reductio: if the view expressed in the formal model allows for such a possibility, then the view is conciliatory in name only. I contend, however, that the view expressed in the model is genuinely conciliatory, in the sense that it preserves the core conciliationist intuition that the only rational response to a genuine disagreement is conciliation. It's just that it is combined with the natural ideas that an agent is typically uncertain about whether or not the disagreement she faces is genuine, and that some beliefs are more rational for an agent to hold than others. And these ideas makes space for cases under discussion. Let's try putting ourselves into the shoes of a committed conciliationist who is more confident in her reasoning about free will than her reasoning about the relevant disagreement:

After thinking hard about the issue of epistemic significance of disagreement, I'm quite sure that conciliation is the only rational response to a genuine disagreement. I've also thought very carefully about the vexed issue of free will, and I'm quite sure that free will exists. My colleague Milo has also worked on free will, and he's told

\footnotetext{
${ }^{51}$ Once Christensen would have been an exception to this claim — see Christensen (2013) but he has modified his view.
} 
me a few times that free will is nonsense - although we haven't been able to find a time to sit down and discuss the question at length. So while I'm fairly confident that we're in genuine disagreement, it's not impossible that our disagreement is merely verbal. If it turned out that it is not merely verbal, I'd immediately suspend judgment on whether free will exists. However, as things stand, I can rationally retain the belief in free will. For I can't say - in good conscience - that Milo's publication record in metaphysics and the few interactions that we have had make me as confident that our disagreement is genuine as my own work makes me confident that free will exists.

I, for my part, think that the agent's train of though here is perfectly reasonable, and, more importantly, that there's no good reason to question her allegiance to conciliationism - at least, if it's kept in mind that, by assumption, the agent's degrees of confidence are rational, or, roughly, in accordance with her evidence. ${ }^{52}$

The second feature that makes the reasoner's response to the free will issue surprising is that it depend on the relative degrees of confidence in the conclusions of the reasoning about free will and the reasoning about conciliationism, Seems $(L)$ and $\operatorname{Seems}(C)$. Most proponents of conciliatory views take on board the idea that the correct doxastic response to a mundane case of disagreement - that is, one in which a committed conciliationist finds out that she's in disagreement over some nontrivial issue with an epistemic peerdepends on how confident the conciliationist is in her take on the issue: the higher her pre-disagreement degree of confidence in her view on $X$, the higher her post-disagreement degree of confidence in her view on $X$ should be. Some proponents of conciliatory views may also accept the idea that the response to mundane cases of disagreement should depend on one's degrees of confidence in conciliationism: the higher one's degree of confidence in conciliationism, the

\footnotetext{
${ }^{52} \mathrm{An}$ anonymous referee remains unconvinced, insisting that the fact that the model allows for cases like the one just discusses shows that the view it captures isn't conciliatory, and, perhaps, that it even leads to a new version of the problem of self-defeat. I'm starting to suspect that it's going to be difficult to find an argument that would convince the referee, relying only on the tools developed in this paper. For, first, my analysis crucially relies on the pre-theoretic idea that the agent is often uncertain about whether or not the disagreement that she's a party to is genuine, and it's not obvious to me that this idea has a natural place in some of the more standard ways of thinking about questions relating to peer disagreement. So if one insists on looking at the cases from a more standard perspective, one is likely to remain dissatisfied with my analysis. And second, the issue appears to be at least in part terminological: if one operates with a stringent definition of conciliationism, one isn't going to call the view captured in the model conciliatory. I'm tempted to think, however, that this shouldn't make the view itself any less interesting - and in spite of the fact that it naturally raises the question about the view's relations to other less-than-conciliatory views from the literature. Also, it's worth adding that the point that conciliationism is compatible with steadfast responses isn't really novel, and that it is often made in the literature. See, for instance, Christensen's (2011) discussion of the case he dubs Careful Checking, a variation of Mental Math in which the agent checks the correctness of her answer multiple times, uses a reliable calculator, and still finds herself in disagreement with the friend - see p. 9ff. Christensen agrees with Lackey $(2010 \mathrm{a}, \mathrm{b})$ and other critics that the rational response to this case is to stick to one's belief, and then goes on to argues that this is compatible with conciliationism.
} 
lower one's post-disagreement degree of confidence in one's view on $X$ should be. Nevertheless the literature proceeds under the assumption that demands that one lowers one's confidence in one's view on $X$, no matter how it compares to one's confidence in conciliationism. ${ }^{53}$ But, as we have seen, it's easy to make intuitive sense of an agent who thinks that conciliationism is correct, finds herself in disagreement with a peer over the question of whether free will exists, and nevertheless retains her belief in the latter: her degree of confidence in the reasoning supporting the conclusion that free will exists is simply higher than her degree of confidence in the reasoning supporting the conclusion that conciliationism is true. So even though our model reasoner's response goes against the orthodoxy, it seems perfectly reasonable.

Thus, the answer to the question of whether or not it is rational to conciliate in a mundane case of disagreement depends on more details than standardly thought. The details that let the agent decide if the disagreement she finds herself in is genuine are important. But so are the details that determine the agent's relative degrees of confidence in conciliationism and her take on the question that's under dispute. In the end, even though the agent holds conciliationism to be true, it's a view on a complex issue she isn't fully certain of, and it's but one among many other views on complex issues she isn't fully certain of. And there doesn't seem to be anything speaking in favor of granting conciliationism hegemony over all these other views.

Finally, the third surprising feature of the reasoner's response to the free will issue is that it does not depend on whether or not conciliationism turns on itself in the scenario - or, to use our notation, on whether $\operatorname{Seems}(C) \leq$ Seems $($ Disagree $(C))$ or, rather, Seems $(C)>\operatorname{Seems}($ Disagree $(C))$ obtains. With few, and mostly recent, exceptions, authors writing on the self-defeat objection have thought that conciliationism's turning on itself either leads, or would lead, to problems having to do specifically with the belief in free will. Ever since Elga (2010) argued that it leads to inconsistent recommendationsto abandon this belief and not to abandon it - most authors have responded to the objection in one of two ways. The majority has tried to argue that conciliationism actually never turns on itself, or that its turning on itself doesn't mean that one has to abandon the belief in it and cease to follow its recommendations. And Matheson (2015a,b) has tried to argue that, whenever conciliationism turns on itself, its recommendation regarding the belief in free will loses its normative force ${ }^{54}$ But all of this literature took it for granted that the answer to the question of what's the correct response regarding the belief in free will depends

\footnotetext{
${ }^{53}$ This claim may need to be hedged to fit all the different views from the literature. Thus, some advocates of conciliationism acknowledge the existence of at least some correlations between one's degrees of confidence in conciliatonism and one's view on some $X$. Matheson $(2015 \mathrm{a}, \mathrm{b})$, in particular, would say that one should retain full confidence in one's view on $X$ in any case where one has overwhelming (misleading) evidence that conciliationism is false. This, however, doesn't change the fact that Matheson takes it for granted that, in typical scenarios, one is to reduce one's confidence in one's view on $X$ in response to a disagreement over $X$, no matter how it compares to one's confidence in conciliationism.

${ }^{54}$ Recall the literature survey from Section 3.4. These are the responses that fall under the first two categories.
} 
on whether or not conciliationism turns on itself.

As we saw in Section 3.4, this dependency has been called into question only recently, and now our analysis provides further evidence against it: it suggests that there are some cases where it's rational to retain the belief in free will and the belief in conciliationism, and that there are others where it's rational to abandon the belief in free will and the belief in conciliationism. ${ }^{55}$ We have already discussed cases fitting the former pattern. Now let's turn to those that fit the latter.

In a typical scenario where the reasoner abandons both the belief in free will and the belief in conciliationism, the degree of confidence in the reasoning leading to the conclusion that free will exists is lower than the degree of confidence in the reasoning leading to the conclusion that conciliationism is correct, $\operatorname{Seems}(L)<\operatorname{Seems}(C)$. (This degree of confidence is also lower than the degree of confidence in the disagreement with Milo being genuine, Seems $(L)<$ Seems (Disagree $(L)$ ), but this latter point is less important for our purposes.) Now, putting ourselves into the shoes of an agent with these degrees of confidence, it seems compelling to reason about the situation as follows:

I've thought about the epistemic significance of disagreement to the best of my ability, and, as far as I can tell, conciliationism is correct. If it is correct, I should back off from the reasoning that led me to conclude that free will exists, since my disagreement with Milo gives me good reason to suspect that this reasoning rests on a mistake. Also, I'm more confident in my reasoning about conciliationism than my reasoning about free will, and yet my disagreement with Evelyn givens me good reason to suspect that the former reasoning rests on a mistake. ${ }^{56}$ Clearly, my conclusion regarding conciliationism is either correct, or it is not. If it is - in spite of the evidence to the contrary - I shouldn't trust my reasoning about free will. And if it is not, then the reasoning that I'm more confident in than my reasoning about free will has led me astray. Should I rely on the reasoning I'm less confident in if the reasoning that I am more confident in turned out to be mistaken? Perhaps, it's safer not to. So, I shouldn't believe that free will exists.

While one might find this train of thought overly cautious, it's perfectly coherent and sensible. So our formal analysis suggests that Littlejohn (2020) is right to think that there isn't necessarily anything wrong with suspending judgment on conciliationism and still conciliating in response to the disagreement over free will. ${ }^{57}$ However, it also suggests that calling the agent's backing off from her belief in free will in such cases conciliating is misleading.

\footnotetext{
${ }^{55}$ You'll recall that both Christensen (2021) and Littlejohn (2020) also suggest that sometimes it can be rational to abandon both beliefs.

${ }^{56}$ The reasoner abandons the belief in conciliationism only in case $\operatorname{Seems}(C) \leq$ Seems(Disagree $(C))$.

${ }^{57}$ See footnote 39 .
} 
The analysis also sheds light on the reasoner's response that we discussed back in Section 3.4, before introducing degrees of confidence. And what it suggests is that we think of the version of Double Disagreement the reasoner was responding to as an atypical case fitting the pattern just discussed. Not making the degrees of confidence explicit in the model amounts to assuming that all the relevant degrees of confidence are equal, $\operatorname{Seems}(L)=\operatorname{Seems}(C)=$ Seems $($ Disagree $(L))=$ Seems $($ Disagree $(L))$. But the above train of thought can be extrapolated to cases where all degrees are equal - although it does become slightly less intuitive. Still, this seems to be enough to conclude that the reasoner's response that looked incoherent to us back in Section 3.4 is, in fact, perfectly reasonable.

Given that our analysis of Double Disagreement readily generalizes to other cases involving disagreements over conciliationism, we have a fully general response to the self-defeat objection. ${ }^{58}$

\section{Conclusion}

Let's take a look back at the distance covered. Our starting point was an important worry about conciliatory views: they would seem to self-defeat and issue inconsistent recommendations in scenarios involving disagreements over their own correctness. Drawing on the work from the defeasible logic paradigm, I devised a model conciliatory reasoner and focused on its behavior in the troublesome scenarios. At first, this reasoner suggested that one is to conclude everything in them, seemingly only reinforcing the worry. However, this was largely due to a technical problem, having to do with the particular framework used. The problem was resolved by moving to the more general framework of argumentation theory. Once modified, the reasoner suggested that, in response to a case where one finds oneself in disagreement over conciliationism, as well as over some other independent question $X$, one is to back off from both one's belief in conciliationism and one's take on $X$. Having noted the seeming incoherence of this response, I went on to suggest that it seems to us incoherent only because the scenario is underdescribed, and that, in particular, what's missing is the information about the reasoning agent's degrees of confidence. In the last third of the paper, I enhanced the model reasoner so that it can take this information into account, and we saw how its responses to the troublesome scenario correlate with the differences in the (relative) degrees of confidence. There were three important upshots. The first was independent of the self-defeat objection: contrary to the

\footnotetext{
${ }^{58}$ Like other responses conceding that conciliationism can self-defeat and that it's rational for one to abandon the belief in it in cases where it self-defeats, my response is committed to a consequence that the advocates of conciliatory views might - perhaps, even should - find embarrassing: in light of the contingent fact that conciliationism currently has many illustrious critics, including Kelly (2005, 2010), Titelbaum (2015), and Wedgwood (2010), it seems very plausible that most advocates of conciliatory views can't hold their views rationally. I've nothing new to say in defense of this unfortunate consequence. However, like other conciliationists, I'm tempted to think that it's a consequence we can live with - see, e.g., (Christensen 2021, 21-2), (Fleisher 2021), (Littlejohn 2013), and (Matheson 2015b, pp. 149-53).
} 
common assumption, a committed conciliationist can rationally retain her view on some issue even if she has good or even excellent evidence suggesting that there's genuine disagreement over it - this is due to the importance of her relative degrees of confidence in conciliationism, in the issue under dispute, and in the disagreement being genuine. The second upshot was that - again, contrary to a widely-held assumption - whether one is to back off from one's take on $X$ in the troublesome scenarios does not depend on whether or not it's rational for one to retain the belief in conciliationism. Notably, this is well in line with the recent ideas of Christensen (2021) and Littlejohn (2020). Finally, the third upshot was that the reasoner's recommendation to adopt the seemingly incoherent doxatic state is perfectly reasonable, given the circumstances it's issued in. So, assuming our model reasoner is true to the spirit of conciliatory views - which, I think, we have all reason to think it is - we have a response to the self-defeat objection against conciliatory views.

Acknowledgment. I would like to thank the audiences at various venues in Amsterdam, Berlin, College Park, New York, and Palo Alto for helpful feedback on earlier versions of the material presented here. I'm also very grateful to the two anonymous referees of this journal for their critical engagement with the paper and their generous and insightful comments, which have lead to many improvements. Finally, I owe a special debt of gratitude to Fabrizio Cariani and John Horty: thanks for the encouragement, for all the advice, for the many discussions, as well as for you detailed comments on earlier drafts.

\section{Appendix}

Observation 2.1. Let $c=\langle\mathcal{W}, \mathcal{R}\rangle$ be an arbitrary regular context where no

Reasonable-formulas occur-or, more precisely, a context where no subformula of any of the formulas in $\mathcal{W}$ or any of the premises or conclusions of the rules in $\mathcal{R}$ is of the form Reasonable $(\mathfrak{r})$. Then there's a context $c^{\prime}=\langle\mathcal{W} \cup\{$ Reasonable $(\mathfrak{r})$ : $r \in \mathcal{R}\}, \mathcal{R}\rangle$ that's equivalent to $c$. Or more explicitly, $X$ follows from $c$ if and only if $X$ follows from $c^{\prime}$ for all $X$ such that no subformula of $X$ is of the form $\operatorname{Reasonable}(\mathfrak{r})$.

Proof. Left-to-right: Take some arbitrary formula $X$ that follows from $c$, according to the original definition of consequence. Then we know that, for every proper scenario $\mathcal{S}$ based on $c$, we have $\mathcal{W} \cup$ Conclusion $[\mathcal{S}] \vdash X$. Now zoom in on one such proper scenario $\mathcal{S}$. By the definition of the notion, for all $r \in \mathcal{S}$, we have $r \in$ Triggered $_{\mathcal{W}, \mathcal{R}}(\mathcal{S}), r \notin$ Conflicted $_{\mathcal{W}, \mathcal{R}}(\mathcal{S})$, and $r \notin \operatorname{Excluded}_{\mathcal{W}, \mathcal{R}}(\mathcal{S})$. Now let's refocus on the new context $c^{\prime}$. It's not difficult to see that $\mathcal{S}$ qualifies as a proper scenario based on $c^{\prime}$. Since all the original information is present in $c^{\prime}$, we can be sure that, for all $r \in \mathcal{S}$, we have $r \in \operatorname{Triggered}_{\mathcal{W}^{\prime}, \mathcal{R}}(\mathcal{S})$, $r \notin$ Conflicted $_{\mathcal{W}^{\prime}, \mathcal{R}}(\mathcal{S})$, and $r \notin \operatorname{Excluded}_{\mathcal{W}^{\prime}, \mathcal{R}}(\mathcal{S})$. What's more, by the construction of $c^{\prime}$, we know that, for every $r \in \mathcal{S}$, there's a formula of the form Reasonable $(\mathfrak{r})$ in the hard information of $c^{\prime}$. Hence, for every $r \in \mathcal{S}$, we have 
$r \in$ Reasonable $_{\mathcal{W}^{\prime}, \mathcal{R}}(\mathcal{S})$. So $\mathcal{S}$ is proper. The same applies to other proper scenarios based on $c$, and so $X$ follows from $c^{\prime}$, according to the modified definition.

Right-to-left: Suppose that $X$ doesn't contain the predicate Reasonable and that $X$ follows from $c^{\prime}$, according to the modified definition of consequence. Then for every proper scenario $\mathcal{S}$ based on $c^{\prime}$, it holds that $\mathcal{W} \cup$ Conclusion $[\mathcal{S}] \vdash$ $X$. Take an arbitrary $\mathcal{S}$. Then, by the definition of proper scenarios, we know that, for all $r \in \mathcal{S}, r \in$ Triggered $_{\mathcal{W}^{\prime}, \mathcal{R}}(\mathcal{S}), r \notin$ Conflicted $_{\mathcal{W}^{\prime}, \mathcal{R}}(\mathcal{S})$, and $r \notin$ Excluded $_{\mathcal{W}^{\prime}, \mathcal{R}}(\mathcal{S})$. By construction, there's no information in $c^{\prime}$ that would have to do with the new predicate and wouldn't be contained in $c$. Hence, for all $r \in \mathcal{S}$, $r \in \operatorname{Triggered}_{\mathcal{W}, \mathcal{R}}(\mathcal{S}), r \notin$ Conflicted $_{\mathcal{W}, \mathcal{R}}(\mathcal{S})$, and $r \notin \operatorname{Excluded}_{\mathcal{W}, \mathcal{R}}(\mathcal{S})$. So $\mathcal{S}$ qualifies as a proper scenario based on $c$, implying that $\mathcal{W} \cup$ Conclusion $[\mathcal{S}] \vdash X$. The same applies to the other proper scenarios based on $c^{\prime}$, and so $X$ follows from $c$, according to the original definition.

Observation 3.1. Let $c=\langle\mathcal{W}, \mathcal{R}\rangle$ be a context and $X$ an arbitrary formula. Then $X$ follows from $c$ in default logic, $c \sim X$, if and only if $X$ follows from $c$ according to stability semantics, $c r_{s} X$.

Proof. Left-to-right: Suppose that $c \sim X$. The, for every proper scenario $\mathcal{S}$ based on $c$, we have $\mathcal{W} \cup$ Conclusion $[\mathcal{S}] \vdash X$. Consider an arbitrary $\mathcal{S}$ of this sort. Since $\mathcal{S} \subseteq$ Reasonable $_{\mathcal{W}, \mathcal{R}}(\mathcal{S})$ and $\mathcal{S} \subseteq \operatorname{Triggered}_{\mathcal{W}, \mathcal{R}}(\mathcal{S})$, the set $\mathcal{S}$ is an element of $\operatorname{Arguments}(c)$. Now we will show that that $\mathcal{S}$ defeats every argument $\mathcal{S}^{\prime}$ in Arguments $(c)$ such that $\mathcal{S}^{\prime} \nsubseteq \mathcal{S}$. So take an arbitrary $\mathcal{S}^{\prime}$ of this sort and consider $\mathcal{S}^{\prime \prime}=\mathcal{S} \cap \mathcal{S}^{\prime}$. Now take some rule $r$ from $\mathcal{S}^{\prime}$ such that $r \in$ Reasonable $_{\mathcal{W}, \mathcal{R}}\left(\mathcal{S}^{\prime \prime}\right), r \in$ Triggere $_{\mathcal{W}, \mathcal{R}}\left(\mathcal{S}^{\prime \prime}\right)$. Such an $r$ has to exist because $\mathcal{S}^{\prime} \in \operatorname{Argument}(c)$ and $\mathcal{S}^{\prime \prime} \subset \mathcal{S}^{\prime}$. In light of the fact that $r \in$ Reasonable $_{\mathcal{W}, \mathcal{R}}\left(\mathcal{S}^{\prime \prime}\right)$ and $r \in \operatorname{Triggered}_{\mathcal{W}, \mathcal{R}}\left(\mathcal{S}^{\prime \prime}\right)$, it has to be the case that $r \in \operatorname{Reasonable}_{\mathcal{W}, \mathcal{R}}(\mathcal{S})$, $r \in$ Triggered $_{\mathcal{W}, \mathcal{R}}(\mathcal{S})$. So, given that $\mathcal{S}$ is a proper scenario, it must be the case that either $r \in \operatorname{Conflicted}_{\mathcal{W}, \mathcal{R}}(\mathcal{S})$ or $r \in \operatorname{Excluded}_{\mathcal{W}, \mathcal{R}}(\mathcal{S})$. So either $\mathcal{W} \cup$ Conclusion $[\mathcal{S}] \vdash \neg$ Conclusion $[r]$ or $\mathcal{W} \cup$ Conclusion $[\mathcal{S}] \vdash \operatorname{Out}(\mathfrak{r})$. But in either case we get $\mathcal{S} \leadsto \mathcal{S}^{\prime}$. Set $\Gamma=\left\{\mathcal{S}^{\prime} \in \operatorname{Arguments}(c): \mathcal{S}^{\prime} \subseteq \mathcal{S}\right\}$. Since $\mathcal{S}$ defeats every $\mathcal{S}^{\prime}$ in Arguments $(c)$, the set of arguments $\Gamma$ defeats every argument that is not contained in $\Gamma$. And given that $\mathcal{S}$ is a proper scenario, $\Gamma$ has to be consistent. So $\Gamma$ is a stable extension of $\mathcal{F}(c)$. What's more, $X$ follows from $\Gamma$, as it contains $\mathcal{S}$ and $\mathcal{W} \cup$ Conclusion $[\mathcal{S}] \vdash X$. Notice that we can run the same argument for every other proper scenario based on $c$. Consequently, $c r_{s} X$.

Right-to-left: Suppose that $c r_{s} X$. This means that, for every stable extension $\Gamma$ of $\mathcal{F}(c)$, it holds that $\Gamma$ contains some argument $\mathcal{S}$ such that $\mathcal{W} \cup$ Conclusion $[\mathcal{S}] \vdash X$. Let's now focus on one such stable extension $\Gamma$.

The first step is to show that this $\Gamma$ has a maximal element, that is, an argument $\mathcal{S}$ such that, for all $\mathcal{S}^{\prime} \in \Gamma$, we have $\mathcal{S}^{\prime} \subseteq \mathcal{S}$. To show that this holds, we use a proof by contradiction. Suppose that there's no single maximal element in $\Gamma$. Now take some $\mathcal{S} \in \Gamma$ such that there's no $\mathcal{S}^{\prime} \in \Gamma$ with $\mathcal{S} \subset \mathcal{S}^{\prime}$. Consider an arbitrary rule $r$ from $\mathcal{R}$ such that $r \notin \mathcal{S}$, but $r \in$ Reasonable $_{\mathcal{W}, \mathcal{R}}(\mathcal{S})$ and $r \in$ Triggered $_{\mathcal{W}, \mathcal{R}}(\mathcal{S})$. Since $\mathcal{S}$ is maximal, $\mathcal{S} \cup\{r\} \notin \Gamma$. And given that $\Gamma$ is 
stable, it must hold that $\Gamma \leadsto \mathcal{S} \cup\{r\}$. So there has to be some argument $\mathcal{S}^{\prime} \in \Gamma$ such that $\mathcal{S}^{\prime} \leadsto \mathcal{S} \cup\{r\}$. The expression $\mathcal{S}^{\prime} \leadsto \mathcal{S} \cup\{r\}$ means that there has to be some rule $r^{\prime}$ in $\mathcal{S} \cup\{r\}$ such that $\mathcal{W} \cup$ Conclusion $\left[\mathcal{S}^{\prime}\right] \vdash \neg$ Conclusion $\left[r^{\prime}\right]$ or $\mathcal{W} \cup$ Conclusion $\left[\mathcal{S}^{\prime}\right] \vdash \neg$ Out $\left(\mathfrak{r}^{\prime}\right)$. However, if the rule $r^{\prime}$ in question is anything but $r$ itself, then we would also have $\mathcal{S}^{\prime} \sim \mathcal{S}$, making $\Gamma$ inconsistent. So the argument $\mathcal{S}^{\prime}$ is such that either $\mathcal{W} \cup$ Conclusion $\left[\mathcal{S}^{\prime}\right] \vdash \neg$ Conclusion $[r]$ or $\mathcal{W} \cup$ Conclusion $\left[\mathcal{S}^{\prime}\right] \vdash \neg O u t(\mathfrak{r})$. Notice that has to be such an argument $\mathcal{S}^{\prime}$ for every $r$ with $r \notin \mathcal{S}$, but $r \in$ Reasonable $_{\mathcal{W}, \mathcal{R}}(\mathcal{S})$ and $r \in \operatorname{Triggered}_{\mathcal{W}, \mathcal{R}}(\mathcal{S})$-if there are any such rules at all.

Now let's zoom in on a different set $\mathcal{S}^{\dagger} \in \Gamma$ such that there's no $\mathcal{S}^{\prime} \in \Gamma$ with $\mathcal{S}^{\dagger} \subset \mathcal{S}$. So $\mathcal{S}^{\dagger} \neq \mathcal{S}$. Consider $\mathcal{S} \cap \mathcal{S}^{\dagger}$. Take the rule $r^{\dagger}$ such that $r^{\dagger} \epsilon$ $\mathcal{S}^{\dagger}, r^{\dagger} \in$ Triggered $_{\mathcal{W}, \mathcal{R}}\left(\mathcal{S} \cap \mathcal{S}^{\dagger}\right)$, and $r^{\dagger} \in \operatorname{Triggered}_{\mathcal{W}, \mathcal{R}}\left(\mathcal{S} \cap \mathcal{S}^{\dagger}\right)$. Since $\mathcal{S}$ and $\mathcal{S}^{\dagger}$ are both in $\operatorname{Arguments}(c)$ and $\mathcal{S}^{\dagger} \nsubseteq \mathcal{S}$, such a rule $r^{\dagger}$ must exist. But given the proof in the previous paragraph, we can be sure that has to be an argument $\mathcal{S}^{\prime} \in \Gamma$ such that either $\mathcal{W} \cup$ Conclusion $\left[\mathcal{S}^{\prime}\right] \vdash \neg$ Conclusion $\left[r^{\dagger}\right]$ or $\mathcal{W} \cup$ Conclusion $\left[\mathcal{S}^{\prime}\right] \vdash \operatorname{Out}\left(\mathfrak{r}^{\dagger}\right)$. Since $r^{\dagger} \in \mathcal{S}^{\dagger}$, we have $\mathcal{S} \leadsto \mathcal{S}^{\dagger}$ which entails, contrary to our assumption, that $\Gamma$ is inconsistent. So $\Gamma$ must have a maximal element after all.

It's not difficult to see that the maximal element of $\Gamma$, call it, $\mathcal{S}$, is such that, for all $\mathcal{S}^{\prime} \in \operatorname{Argument}(c)$ with $\mathcal{S}^{\prime} \nsubseteq \mathcal{S}$, it holds that $\mathcal{S} \leadsto \mathcal{S}^{\prime}$. Consider some $\mathcal{S}^{\prime}$ that fits the description. Given that $\Gamma$ is stable, we know that $\Gamma \sim \mathcal{S}^{\prime}$. So there's some argument $\mathcal{S}^{\prime \prime} \in \Gamma$ such that $\mathcal{S}^{\prime \prime} \leadsto \mathcal{S}^{\prime}$, meaning that $\mathcal{W} \cup$ Conclusion $\left[\mathcal{S}^{\prime \prime}\right] \vdash$ $\neg$ Conclusion $[r]$ or $\mathcal{W} \cup$ Conclusion $\left[\mathcal{S}^{\prime \prime}\right] \vdash \operatorname{Out}(\mathfrak{r})$ for some rule $r \in \mathcal{S}^{\prime}$. But since $\mathcal{S}$ is maximal, it must be the case that $\mathcal{W} \cup$ Conclusion $[\mathcal{S}] \vdash \neg$ Conclusion $[r]$ or $\mathcal{W} \cup$ Conclusion $[\mathcal{S}] \vdash \operatorname{Out}(\mathfrak{r})$ for some rule $r \in \mathcal{S}^{\prime}$. Consequently, $\mathcal{S} \leadsto \mathcal{S}^{\prime}$. Another thing that should be clear is that $\mathcal{W} \cup$ Conclusion $[\mathcal{S}] \vdash X$ : If there's an argument in $\Gamma$ that lets us conclude $X$, then $X$ follows from the maximal element too.

The final step is to show that the maximal element $\mathcal{S}$ of $\Gamma$ is a proper scenario based on $c$. What we need to establish, then, is that $\mathcal{S}=\mathcal{S}^{\prime}$ where

$$
\begin{aligned}
\mathcal{S}^{\prime}=\{r \in \mathcal{R}: \quad & r \in \text { Reasonable }_{\mathcal{W}, \mathcal{R}}(\mathcal{S}), \\
& r \in \text { Triggered }_{\mathcal{W}, \mathcal{R}}(\mathcal{S}), \\
& r \notin \text { Conflicted }_{\mathcal{W}, \mathcal{R}}(\mathcal{S}), \\
& \left.r \notin \text { Excluded }_{\mathcal{W}, \mathcal{R}}(\mathcal{S})\right\} .
\end{aligned}
$$

$\mathcal{S} \subseteq \mathcal{S}^{\prime}$ : Take an arbitrary $r$ from $\mathcal{S}$. Since $\mathcal{S}$ is in Argument $(c)$, we know that $r \in$ Reasonsable $_{\mathcal{W}, \mathcal{R}}(\mathcal{S})$ and $r \in \operatorname{Triggered}_{\mathcal{W}, \mathcal{R}}(\mathcal{S})$. Suppose we had $r \in$ Conflicted $_{\mathcal{W}, \mathcal{R}}(\mathcal{S})$. In that case, $\mathcal{S}$ would self-defeat, and $\Gamma$ couldn't be a stable extension. So $r \notin \operatorname{Conflicted}_{\mathcal{W}, \mathcal{R}}(\mathcal{S})$. Now suppose we had $r \epsilon$ $\operatorname{Excluded}_{\mathcal{W}, \mathcal{R}}(\mathcal{S})$. Again, $\mathcal{S}$ would self-defeat, and $\Gamma$ couldn't be a stable extension. So $r \notin$ Excluded $_{\mathcal{W}, \mathcal{R}}(\mathcal{S})$.

$\mathcal{S}^{\prime} \subseteq \mathcal{S}$ : Take an arbitrary $r$ such that $r$ is an element of $\operatorname{Reasonable}_{\mathcal{W}, \mathcal{R}}(\mathcal{S})$ and $\operatorname{Triggered}_{\mathcal{W}, \mathcal{R}}(\mathcal{S})$ and $r$ is not an element of either $\operatorname{Conflicted}_{\mathcal{W}, \mathcal{R}}(\mathcal{S})$, or $\operatorname{Excluded}_{\mathcal{W}, \mathcal{R}}(\mathcal{S})$. Now suppose, toward a contradiction, that $r \notin \mathcal{S}$. Let $\mathcal{S}^{\dagger}=\mathcal{S} \cup\{r\}$. Since $\mathcal{S}^{\dagger} \subseteq$ Triggered $_{\mathcal{W}, \mathcal{R}}\left(\mathcal{S}^{\dagger}\right)$ and $\mathcal{S}^{\dagger} \subseteq$ Reasonable $_{\mathcal{W}, \mathcal{R}}\left(\mathcal{S}^{\dagger}\right), \mathcal{S}^{\dagger}$ 
must be in $\operatorname{Argument}(c)$. What's more, we do not have $\mathcal{S} \leadsto \mathcal{S}$, and so $\Gamma \boldsymbol{\psi}^{\dagger} \mathcal{S}^{\dagger}$. This is enough to conclude that $\Gamma$ is not a stable extension after all.

This shows that $\mathcal{S}$ is proper. Since we can run the same argument for every other stable extension, we know that $c \sim X$.

Observation 3.2. Let $c=\langle\mathcal{W}, \mathcal{R}\rangle$ be a context and $\mathcal{F}(c)=\langle\mathcal{A}, \leadsto\rangle$ an argument framework constructed from it. If $\mathcal{F}(c)$ does not contain either odd cycles of defeat or infinite chains of defeat, then $c \sim_{s} X$ if and only if $c r_{p} X$.

Proof. We will show that an argument set $\Gamma$ is a stable extension of $\mathcal{F}(c)$ if and only if it is a preferred extensions of $\mathcal{F}(c)$. The result follows immediately.

Left-to-right (Dung 1995): This direction holds independently of the assumption. Let $\Gamma$ be a stable extension of $\mathcal{F}(c)$. So, for all $\mathcal{S} \in \mathcal{A} \backslash \Gamma, \Gamma \sim \mathcal{S}$. It's easy to see that $\Gamma$ is complete: Consider an argument $\mathcal{S}$ such that $\Gamma$ defends $\mathcal{S}$. If $\mathcal{S} \in \Gamma$, we're done. So suppose $\mathcal{S} \notin \Gamma$. Since $\Gamma$ is stable, we have it that $\Gamma \leadsto \mathcal{S}$. Thus, there's an argument $\mathcal{S}^{\prime} \in \Gamma$ such that $\mathcal{S}^{\prime} \leadsto \mathcal{S}$. Given that $\Gamma$ defends $\mathcal{S}$, there has to be an $\mathcal{S}^{\dagger}$ in $\Gamma$ such that $\mathcal{S}^{\dagger} \leadsto \mathcal{S}^{\prime}$. But this would mean that $\Gamma$ is not conflict-free, which contradicts it being stable. Now let's verify that $\Gamma$ is not only a complete extension, but also a maximal complete extensions: Suppose that it wasn't. There would be another complete extension $\Gamma^{\prime}$ such that $\Gamma \subset \Gamma^{\prime}$. Let $\mathcal{S}$ be an argument such that $\mathcal{S} \notin \Gamma$ and $\mathcal{S} \in \Gamma^{\prime}$. Since $\Gamma$ is stable, $\Gamma \leadsto \mathcal{S}$, and so $\Gamma^{\prime} \leadsto \mathcal{S}$. Then, however, $\Gamma^{\prime}$ is not conflict-free, which contradicts it being complete.

Right-to-left: Suppose that $\Gamma$ is a preferred, but not a stable extension of $\mathcal{F}(c)$. So $\Gamma$ is a maximal complete extension, and yet there is some argument $\mathcal{S}_{1} \in \mathcal{A}$ such that $\mathcal{S}_{1} \notin \Gamma$ and $\Gamma \rightsquigarrow \mathcal{S}_{1}$. Since $\Gamma$ is complete, it can't defend $\mathcal{S}_{1}$. So there has to be an argument $\mathcal{S}_{2} \in \mathcal{A}$ such that $\mathcal{S}_{2} \notin \Gamma, \mathcal{S}_{2} \leadsto \mathcal{S}_{1}$, and $\Gamma \gg \mathcal{S}_{2}$. And then, again, given that $\Gamma$ is complete, it can't defend $\mathcal{S}_{2}$. So there has to be an argument $\mathcal{S}_{3} \in \mathcal{A}$ such that $\mathcal{S}_{3} \notin \Gamma, \mathcal{S}_{3} \leadsto \mathcal{S}_{2}$, and $\Gamma \rtimes \mathcal{S}_{3}$. We can apply the same line of reasoning to $\mathcal{S}_{4}$ and further, but eventually it has to stop, since, by assumption, there are no infinitely ascending chains of defeat in $\mathcal{F}(c)$. So we will end up with the following possibly very long, but finite chain:

$$
\mathcal{S}_{n} \leadsto \mathcal{S}_{n-1} \leadsto \cdots \leadsto \mathcal{S}_{3} \leadsto \mathcal{S}_{2} \leadsto \mathcal{S}_{1},
$$

and we will have established on the way that, for all $i$ with $1 \leq i<n, S_{i} \notin \Gamma$. Call the set containing the arguments in this chain $\Delta$. Now there are two possibilities: either $\mathcal{S}_{n}=\mathcal{S}_{j}$ for some $1 \leq j<n$ or not. If the latter, no arguments in $\mathcal{A}$ defeats $\mathcal{S}_{n}$. This implies that $\mathcal{S}_{n}$ is defended by $\Gamma$, and, in light of $\Gamma$ being complete, that $\mathcal{S}_{n} \in \Gamma$. So we have a contradiction showing that every defeat chain between arguments from $\mathcal{A}$ that aren't in $\Gamma$ has to end in a cycle. In particular, we have $\mathcal{S}_{n}=\mathcal{S}_{j}$ for some $1 \leq j<n$. What's more, $\mathcal{S}_{j} \leadsto \mathcal{S}_{n-1} \leadsto \cdots \leadsto \mathcal{S}_{j+1} \leadsto \mathcal{S}_{j}$ must be an even cycle - since, by assumption, $\mathcal{F}(c)$ doesn't contain any odd cycles of defeat.

Now let $\Delta^{\prime}=\left\{\mathcal{S}_{i} \in \Delta: i=n-2 k\right.$ with $\left.k \in \mathbb{N}\right\}$ and $\Delta^{\prime \prime}=\left\{\mathcal{S}_{i} \in \Delta: i=\right.$ $n-(2 k+1)$ with $k \in \mathbb{N}\}$. Notice that $\Delta^{\prime}$ is consistent, and that it defends all of 
its arguments from $\Delta^{\prime \prime}$. If $\Delta^{\prime}$ was inconsistent, there would be and odd cycle of defeat in $\mathcal{F}(c)$ after all. And if $\Delta^{\prime}$ didn't defend one of its arguments from $\Delta^{\prime \prime}$, $\mathcal{S}_{n} \leadsto \mathcal{S}_{n-1} \leadsto \cdots \leadsto \mathcal{S}_{2} \leadsto \mathcal{S}_{1}$ couldn't be a chain ending in a cycle.

Next, consider some arbitrary argument $\mathcal{S}^{\dagger}$ from $\Delta^{\prime}$. It's in principle possible that $\mathcal{A}$ contains an argument $\mathcal{S}_{1}^{\prime}$ such that $\mathcal{S}_{1}^{\prime} \sim \mathcal{S}^{\dagger}$ and $\mathcal{S}_{1}^{\prime} \notin \Delta^{\prime \prime}$. Above we have already established that $\mathcal{S}_{1}^{\prime} \notin \Gamma$. And, given that we have also established that every chain of defeat between arguments from $\mathcal{A}$ that are not in $\Gamma$ has to end in an even cycle, we can be sure that the chain

$$
\mathcal{S}_{m}^{\prime} \leadsto \cdots \leadsto \mathcal{S}_{1}^{\prime} \leadsto \mathcal{S}^{\dagger} \leadsto \cdots \leadsto \mathcal{S}_{2} \leadsto \mathcal{S}_{1}
$$

ends in an even cycle too. Notice that it's possible that $\mathcal{S}_{m}^{\prime}=\mathcal{S}_{i}$ for some $1 \leq i \leq n$ (where the $\mathcal{S}_{i}$ is also in $\Delta^{\prime}$, see below). Let $\Theta=\left\{\mathcal{S}_{1}^{\prime}, \ldots, \mathcal{S}_{m}^{\prime}\right\}$ and $\Theta^{\prime}=\left\{S_{i}^{\prime} \in \Theta: i=2 k\right.$ with $2 k \in \mathbb{N}$ and $\left.1<i \leq m\right\}$. It's not difficult to see that the set $\Delta^{\prime} \cup \Theta^{\prime}$ defends $\mathcal{S}^{\dagger}$ and also that $\Delta^{\prime} \cup \Theta^{\prime}$ defends all of the arguments in it from the set $\Delta^{\prime \prime} \cup \Theta^{\prime \prime}$ where $\Theta^{\prime \prime}=\Theta \backslash \Theta^{\prime}$. This follows from what we already know about the relation between $\Delta^{\prime}$ and $\Delta^{\prime \prime}$ and the fact that $\mathcal{S}_{m}^{\prime} \leadsto \cdots \leadsto \mathcal{S}_{1}^{\prime}$ ends in a cycle. Now it remains to show that $\Delta^{\prime} \cup \Theta^{\prime}$ is disjoint from $\Delta^{\prime \prime}$ (and so consistent). So suppose, toward a contradiction, that it's not. Then there has to be some argument $\mathcal{S}^{\ddagger}$ such that $\mathcal{S}^{\ddagger} \in \Theta^{\prime}$ and $\mathcal{S}^{\ddagger} \in \Delta^{\prime \prime}$ (clearly, $\Delta^{\prime}$ and $\Delta^{\prime \prime}$ are disjoint). Given how we defined $\Theta^{\prime}$, it's clear that the chain $\mathcal{S}^{\ddagger} \leadsto \cdots \leadsto \mathcal{S}_{1}^{\prime} \leadsto \mathcal{S}^{\dagger}$ is of even length. Further, given that $\mathcal{S}^{\ddagger} \in \Delta$, a subchain of $\mathcal{S}_{n} \leadsto \cdots \leadsto \mathcal{S}_{2} \leadsto \mathcal{S}_{1}$ has to connect $\mathcal{S}^{\dagger}$ to $\mathcal{S}^{\ddagger}$. Since $\mathcal{S}^{\dagger}$ is in $\Delta^{\prime}$ and $\mathcal{S}^{\ddagger}$ is in $\Delta^{\prime \prime}$, this chain has to be of odd length. But the two chains that connect $\mathcal{S}^{\dagger}$ and $\mathcal{S}^{\ddagger}$ form a cycle, and, since one of them is of even and the other of odd length, we get a contradiction: $\mathcal{F}(c)$ contains an odd cycle of defeat. Thus, $\Delta^{\prime} \cup \Theta^{\prime}$ has to be disjoint from $\Delta^{\prime \prime}$.

Notice that we have effectively shown how to extend $\Delta^{\prime}$ into a larger set of arguments $\Delta^{\prime} \cup \Theta^{\prime}$ that defends $\mathcal{S}^{\dagger}$ from a defeat that doesn't come from $\Delta^{\prime \prime}$ (if there's one). Since $\mathcal{S}^{\dagger}$ was chosen arbitrary, the same line of reasoning can be applied to any argument from $\Delta^{\prime}$ and the larger set $\Delta^{\prime} \cup \Theta^{\prime}$.

Now let's assume, without loss of generality, that there is no argument $\mathcal{S}$ in $\mathcal{A}$ such that $\mathcal{S} \leadsto \Delta^{\prime} \cup \Theta^{\prime}$ and $\mathcal{S} \notin \Delta^{\prime \prime} \cup \Theta^{\prime \prime}$. Let $\Gamma^{\prime}=\Gamma \cup \Delta^{\prime} \cup \Theta^{\prime}$. Clearly, $\Gamma^{\prime}$ is a consistent and a complete extension of $\mathcal{F}(c)$. The facts that $\Gamma^{\prime}$ is complete and that $\Gamma \subset \Gamma^{\prime}$ imply that $\Gamma$ is not a maximal complete extension after all. Thus, we get a contradiction and can conclude that $\Gamma$ has to be a stable extension.

Observation 3.3. Let $c=\langle\mathcal{W}, \mathcal{R}\rangle$ be a context and $\mathcal{S}$ and $\mathcal{S}^{\prime}$ arguments from the argument framework $\mathcal{F}(c)$ constructed from it. Then $\mathcal{S}$ defeats $\mathcal{S}^{\prime}$, according to Definition 11, $\mathcal{S} \leadsto \mathcal{S}^{\prime}$, if and only if $\mathcal{S}$ defeats $\mathcal{S}^{\prime}$, according to Definition 21, $\mathcal{S} \sim_{a} \mathcal{S}^{\prime}$.

Proof. Right-to-left: Suppose that $\mathcal{S} \sim_{a} \mathcal{S}^{\prime}$. This implies that there are arguments $\mathcal{S}^{\dagger}$ and $\mathcal{S}^{\ddagger}$ in the set $\operatorname{Arguments}(c)$ such that $\mathcal{S}^{\dagger} \subseteq \mathcal{S}, \mathcal{S}^{\ddagger} \subseteq \mathcal{S}^{\prime}$, and $\mathcal{S}^{\dagger} \sim_{b} \mathcal{S}^{\ddagger}$. From here, either $\mathcal{W} \cup$ Conclusion $\left[\mathcal{S}^{\dagger}\right] \vdash \neg$ Conclusion $[r]$ or $\mathcal{W} \cup$ Conclusion $\left[\mathcal{S}^{\dagger}\right] \vdash \operatorname{Out}(\mathfrak{r})$ for some rule $r$ from $\mathcal{S}^{\ddagger}$. Since $\mathcal{S}^{\dagger} \subseteq \mathcal{S}$ and $\mathcal{S}^{\ddagger} \subseteq \mathcal{S}^{\prime}$, it follows that $\mathcal{W} \cup$ Conclusion $[\mathcal{S}] \vdash \neg$ Conclusion $[r]$ or $\mathcal{W} \cup$ Conclusion $[\mathcal{S}] \vdash$ $\operatorname{Out}(\mathfrak{r})$ for some rule $r$ from $\mathcal{S}^{\prime}$. And this is enough to conclude that $\mathcal{S} \leadsto \mathcal{S}^{\prime}$. 
Left-to-right: Suppose that $\mathcal{S} \leadsto \mathcal{S}^{\prime}$. This means that there is a rule $r \in \mathcal{S}^{\prime}$ such that either $\mathcal{W} \cup$ Conclusion $[\mathcal{S}] \vdash \neg$ Conclusion $[r]$, or $\mathcal{W} \cup$ Conclusion $[\mathcal{S}] \vdash$ $\operatorname{Out}(\mathfrak{r})$. Without loss of generality, suppose that $\mathcal{W} \cup$ Conclusion $[\mathcal{S}] \vdash \operatorname{Out}(\mathfrak{r})$. Now take the (set-theoretically) smallest argument $\mathcal{S}^{\dagger}$ in $\operatorname{Arguments}(c)$ such that $\mathcal{W} \cup$ Conclusion $\left[\mathcal{S}^{\dagger}\right] \vdash \operatorname{Out}(\mathfrak{r})$ and $\mathcal{S}^{\dagger} \subseteq \mathcal{S}$. Since $\mathcal{S}$ is in $\operatorname{Arguments}(c)$, we know that $\mathcal{S}^{\dagger}$ exists. It's easy to see that $\mathcal{S}^{\dagger}$ is in the set $\operatorname{Minimal}_{\mathcal{F}(c)}(\operatorname{Out}(\mathfrak{r}))$ : If not, then there must be another set $\mathcal{S}^{\ddagger} \subset \mathcal{S}^{\dagger}$ in $\operatorname{Arguments}(c)$ such that $\mathcal{W} \cup$ Conclusion $\left[\mathcal{S}^{\ddagger}\right] \vdash \operatorname{Out}(\mathfrak{r})$. In that case, however, we'd also have $\mathcal{S}^{\ddagger} \subset \mathcal{S}$, contradicting our assumption that $\mathcal{S}^{\dagger}$ is the smallest arguments that's also a subset of $\mathcal{S}$ that entails $\operatorname{Out}(\mathfrak{r})$ with $\mathcal{W}$. Given our definition of basic defeat, $\mathcal{S}^{\dagger} \sim_{b} \mathcal{S}^{\prime \prime}$, for any $\mathcal{S}^{\prime \prime}$ such that $\mathcal{S}^{\prime \prime}$ is in $\operatorname{Minimal}_{\mathcal{F}(c)}(r)$. Let $\mathcal{S}^{\ddagger}$ be the (settheoretically) smallest argument from Arguments $(c)$ with both $r \in \mathcal{S}^{\ddagger}$ and $\mathcal{S}^{\ddagger} \subseteq$ $\mathcal{S}^{\prime}$. Since $\mathcal{S}^{\prime}$ is in Arguments $(c)$, we can be sure that $\mathcal{S}^{\ddagger}$ exists. It's again easy to see that $\mathcal{S}^{\ddagger}$ is among the arguments in $\operatorname{Minimal}_{\mathcal{F}(c)}(r)$, from which it follows that $\mathcal{S}^{\dagger} \sim_{b} \mathcal{S}^{\ddagger}$. Finally, given that $\mathcal{S}^{\dagger} \subseteq \mathcal{S}$ and $\mathcal{S}^{\ddagger} \subseteq \mathcal{S}^{\prime}$, we also have $\mathcal{S} \gg_{a} \mathcal{S}^{\prime}$.

Observation 4.1. Let $c=\langle\mathcal{W}, \mathcal{R}\rangle$ be a regular context and $c^{\prime}=\langle\mathcal{W}, \mathcal{R}, \leq\rangle$ be the same context with a connected preorder $\leq$ assigning all the rules $r$ in $\mathcal{R}$ the same weight-so, for all $r, r^{\prime} \in \mathcal{R}, r \sim r^{\prime}$. Then $\mathcal{F}(c)=\mathcal{F}\left(c^{\prime}\right)$.

Proof. The sets of arguments of $\mathcal{F}(c)$ and $\mathcal{F}\left(c^{\prime}\right)$ are clearly the same. So it remains to show that the defeat relations among the arguments in them coincide.

Left-to-right: Take two arbitrary arguments $\mathcal{S}$ and $\mathcal{S}^{\prime}$ from $\mathcal{F}(c)$ with $\mathcal{S} \leadsto$ $\mathcal{S}^{\prime}$. Since $\mathcal{S} \leadsto \mathcal{S}^{\prime}$, we know that there's some rule $r$ in $\mathcal{S}^{\prime}$ such that either $\mathcal{W} \cup$ Conclusion $[\mathcal{S}] \vdash \neg$ Conclusion $[r]$, or $\mathcal{W} \cup$ Conclusion $[\mathcal{S}] \vdash$ Out $(\mathfrak{r})$. Now, the fact that all rules $\mathcal{R}$ are assigned the same weights immediately entails that $r \leq r^{\prime}$, for all $r^{\prime} \in \mathcal{S}$, which is enough to conclude that $\mathcal{S}^{\prime} \leq \mathcal{S}$. And thus, $\mathcal{S} \leadsto \leq \mathcal{S}^{\prime}$.

Right-to-left: Take two arbitrary arguments $\mathcal{S}$ and $\mathcal{S}^{\prime}$ from $\mathcal{F}\left(c^{\prime}\right)$ such that $\mathcal{S} \leadsto \leq \mathcal{S}^{\prime}$. Since $\mathcal{S} \leadsto \leq \mathcal{S}^{\prime}$, we know that there's some rule $r$ in $\mathcal{S}^{\prime}$ such that either $\mathcal{W} \cup$ Conclusion $[\mathcal{S}] \vdash \neg$ Conclusion $[r]$, or $\mathcal{W} \cup$ Conclusion $[\mathcal{S}] \vdash \operatorname{Out}(\mathfrak{r})$. And this is enough to conclude that $\mathcal{S} \leadsto \mathcal{S}^{\prime}$.

\section{References}

Barberà, S., Bossert, W., \& Pattaniak, P. (2004). Ranking sets of objects. In S. Barberà, P. Hammond, \& C. Seidl (Eds.), Handbook of Utility Theory, Volume 2 (pp. 893-977). Springer Science+Business Media.

Barringer, H., Gabbay, D., \& Woods, J. (2012). Temporal, numerical and metalevel dynamics in argumentation networks. Argument and Computation, 3(23), 143-202.

Bogardus, T. (2009). A vindication of the equal-weight view. Episteme, 6(3), $324-35$. 
Brass, S. (1991). Deduction with supernormal defaults. In G. Brewka, K. Jantke, \& P. Schmitt (Eds.), Nonmonotonic and Inductive Logics. Lecture Notes in Computer Science, Volume 659 (pp. 153-74).: Springer.

Christensen, D. (2007). Epistemology of disagreement: The good news. Philosophical Review, 116, 187-217.

Christensen, D. (2010). Higher-order evidence. Philosophy and Phenomenological Research, 81(1), 185-215.

Christensen, D. (2011). Disagreement, question-begging and epistemic selfcriticism. Philosophers' Imprint, 11(6), 1-22.

Christensen, D. (2013). Epistemic modesty defended. In D. Christensen \& J. Lackey (Eds.), The Epistemology of Disagreement: New Essays (pp. 77-97). Oxford University Press.

Christensen, D. (2016). Conciliation, uniqueness and rational toxicity. Noûs, $50(3), 584-603$.

Christensen, D. (2021). Akratic (epistemic) modesty. Philosophical Studies, $178,2191-214$.

Conee, E. \& Feldman, R. (2004). Evidentialism: Essays in Epistemology. Oxford University Press.

Decker, J. (2014). Conciliation and self-incrimination. Erkenntnis, 79, 1099-134.

Dung, P. M. (1995). On the acceptability of arguments and its fundamental role in nonmonotonic reasoning, logic programming and n-person games. Artificial Intelligence, $77(2), 321-57$.

Dunne, P., Hunter, A., McBurney, P., Parsons, S., \& Wooldridge, M. (2011). Weighted argument systems: Basic definitions, algorithms, and complexity results. Artificial Intelligence, 175(2), 457-86.

Elga, A. (2007). Reflection and disagreement. Nô̂s, 41(3), 478-502.

Elga, A. (2010). How to disagree about how to disagree. In R. Feldman \& T. Warfield (Eds.), Disagreement (pp. 175-86). Oxford University Press.

Feldman, R. (2005). Respecting the evidence. Philosophical Perspectives, 19(1), 95-119.

Feldman, R. (2006). Epistemological puzzles about disagreement. In S. Hetherington (Ed.), Epistemology Futures (pp. 216-36). Oxford University Press.

Feldman, R. (2009). Evidentialism, higher-order evidence, and disagreement. Episteme, 6, 294-312.

Fleisher, W. (2021). How to endorse conciliationism. Synthese, 198, 9913-39. 
Gabbay, D. (2012). An equational approach to argumentation networks. Argument and Computation, 3(2-3), 87-142.

Gelfert, A. (2011). Who is an epistemic peer? Logos and Episteme, 2(4), 507-14.

Grossi, D. \& Modgil, S. (2015). On the graded acceptability of arguments. In Proceedings of the 24th Joint Conference on Artificial Intelligence, IJCAI (pp. 868-74).: AAAI Press.

Horty, J. F. (2012). Reasons as Defaults. Oxford University Press.

Kelly, T. (2005). The epistemic significance of disagreement. Oxford Studies in Epistemology, 1, 179-92.

Kelly, T. (2010). Peer disagreement and higher-order evidence. In R. Feldman \& T. Warfield (Eds.), Disagreement (pp. 111-74). Oxford University Press.

King, N. (2012). Disagreement: What's the problem? Or a good peer is hard to find. Philosophy and Phenomenological Research, 85(2), 249-72.

Lackey, J. (2010a). A justificationists view of disagreement's epistemic significance. In A. Haddock, A. Millar, \& D. Pritchard (Eds.), Social Epistemology (pp. 298-325). Oxford University Press.

Lackey, J. (2010b). What should we do when we disagree? Oxford Studies in Epistemology, 3, 274-93.

Lasonen-Aarnio, M. (2013). Disagreement and evidential attenuation. Noûs, 47, 767-94.

Littlejohn, C. (2013). Disagreement and defeat. In D. Machuca (Ed.), Disagreement and Skepticism (pp. 169-92). Routledge.

Littlejohn, C. (2020). Should we be dogmatically conciliatory? Philosophical Studies, 177, 1381-98.

Matheson, J. (2015a). Are conciliatory views of disagreement self-defeating? Social Epistemology, 29(2), 145-59.

Matheson, J. (2015b). The Epistemic Significance of Disagreement. Pelgrave Macmillan.

Matheson, J. (2018). Disagreement and epistemic peers. In Oxford Handbooks Online. DOI: 10.1093/oxfordhb/97801999353.

Modgil, S. \& Prakken, H. (2013). A general account of argumentation with preferences. Artificial Intelligence, 195, 361-97.

Pittard, J. (2015). Resolute conciliationism. The Philosophical Quarterly, $65(260), 442-63$.

Pollock, J. (1994). Justification and defeat. Artificial Intelligence, 67, 377-407. 
Pollock, J. (1995). Cognitive Carpentry: A Blueprint for How to Build a Person. Cambridge, MA: MIT Press.

Pollock, J. (2001). Defeasible reasoning with variable degrees of justification. Artificial Intelligence, 133, 233-82.

Pollock, J. (2009). A recursive semantics for defeasible reasoning. In G. Simari \& I. Rahwan (Eds.), Argumentation in Artificial Intelligence (pp. 173-97). Springer.

Pollock, J. (2010). Defeasible reasoning and degrees of justification. Argument and Computation, 1(1), 7-22.

Prakken, H. \& Sartor, G. (1997). Argument-based extended logic programming with defeasible priorities. Journal of Applied Non-Classical Logic, 1(2), 25-75.

Prakken, H. \& Vreeswijk, G. (2001). Logics for defeasible argumentation. In D. M. Gabbay \& F. Guenthner (Eds.), Handbooks of Philosophical Logic, volume 4. Springer, Dordrecht.

Reiter, R. (1980). A logic for default reasoning. Artificial Intelligece, 13, 81-132.

Titelbaum, M. (2015). Rationality's fixed point (or: in defense of right reason). Oxford Studies in Epistemology, 5, 253-94.

Weatherson, B. (2013). Disagreements, philosophical, and otherwise. In D. Christensen \& J. Lackey (Eds.), The Epistemology of Disagreement: New Essays (pp. 54-73). Oxford University Press.

Wedgwood, R. (2010). The moral evil demons. In R. Feldman \& T. Warfield (Eds.), Disagreement (pp. 216-46). Oxford University Press. 